\title{
Probabilistic approach to rock physics modeling
}

\author{
Dario Grana ${ }^{1}$
}

\begin{abstract}
Rock physics modeling aims to provide a link between rock properties, such as porosity, lithology, and fluid saturation, and elastic attributes, such as velocities or impedances. These models are then used in quantitative seismic interpretation and reservoir characterization. However, most of the geophysical measurements are uncertain; therefore, rock physics equations must be combined with mathematical tools to account for the uncertainty in the data. We combined probability theory with rock physics modeling to make predictions of elastic properties using probability distributions rather than definite values. The method provided analytical solutions of rock physics models in which the input is a random variable whose exact value is unknown but whose probability distribution is known. The probability distribution derived with this approach can be used to quantify the uncertainty in rock physics model predictions and in rock property estimation from seismic attributes. Examples of fluid substitution and rock physics modeling were studied to illustrate the application of the method.
\end{abstract}

\section{INTRODUCTION}

Rock physics addresses the relationships between rock properties, such as porosity, lithology, and fluid saturations, and elastic properties, such as velocities or impedances. A rock physics model is then a set of equations that transforms the rock properties into elastic attributes. The model can be a simple empirical relation that fits well-log observations or a more sophisticated theoretical model, such as granular media models or inclusion models (Mavko et al., 2009). The model can depend on a single parameter, for example, porosity, or it can involve multiple properties, for example, porosity, clay content, water, and oil saturations of a rock. The common feature of all these models is that the inputs are assigned values. In other words, when we apply a rock physics model, we generally assume that the input data are correct. However, well-log data and laboratory measurements are all affected by errors that depend on the precision of the tools used for the measurements and also on the natural variability and heterogeneity of the rock. Furthermore, most of the measurements we use in rock physics are not actual measurements, but the result of another mathematical operation. For example, the well curves of porosity, clay content, and water saturation are the results of formation evaluation analysis in which actual measurements (gamma ray, neutron porosity, density, and resistivity) are transformed into the above-mentioned petrophysical curves.

In oil recovery, it is essential to evaluate the uncertainty associated to each step of the reservoir modeling workflow. Even if rock physics is a very accurate approximation of the reality, the input data are generally uncertain; therefore, the rock physics model predictions are uncertain as well. We propose here a new probabilistic approach to rock physics modeling in which the input data are not assigned definite values but probability distributions. As a consequence, the result of the rock physics model is not just a prediction with a single value but a probability distribution that will show the most likely value of our prediction and also the associated uncertainty.

A probability distribution assigns a probability value to each measurable subset of the possible outcomes of a random variable (Papoulis, 1984). For example, if the well log indicates that at a given depth, porosity is $30 \%$ but we know that due to the tool precision the measurement error is $\pm 1 \%$, then instead of using the measured value of $30 \%$, we should use a probability distribution, normal or triangular, with mean $30 \%$ and choose the variance such that it will describe the uncertainty in the measurement. Another example is for gas saturation: We know from rock physics measurements that a small amount of gas, such as $10 \%$, largely affects velocities. However, it is hard to distinguish between a small amount of gas and a large amount of gas; therefore, we should use a uniform probability distribution between $10 \%$ and $90 \%$ to describe the gas saturation distribution rather than use a single value.

A similar concept has already been presented in the past in statistical rock physics. Statistical rock physics is introduced by Mavko 
and Mukerji (1998), where they combine rock physics modeling with Monte Carlo simulations to generate multiple geologic scenarios to be used in hydrocarbon prospect detection from seismic data. The technique consists in assuming a distribution of a given rock or fluid property, generating a set of samples, applying the rock physics model, and computing a training data set of rock physics predictions to be used as a catalog for seismic interpretation. Similar strategies are presented later in Avseth et al. (2001) and Mukerji et al. (2001), where the authors first create a training data set of rock physics model predictions and then use this training data set to classify inverted seismic attributes. A good description of this technique with multiple examples is given in Avseth et al. (2005). The same statistical rock physics approach has then been adopted in several inversion workflows. Indeed, the physical model can be any rock physics model: velocity-saturation (Mavko and Mukerji, 1998), velocity-porosity (Doyen, 1988), velocity-pressure (Doyen, 2007), amplitudes-velocities (Buland and Omre, 2003), and porosity-permeability, etc.; it can include discrete properties such as facies classification (Eidsvik et al., 2004; Gallop, 2006), and it can involve multiple properties (Bachrach, 2006; Larsen et al., 2006; Gunning and Glinsky, 2007; Buland et al., 2008; González et al., 2008; Spikes et al., 2008; Bosch et al., 2009; Grana and Della Rossa, 2010; Rimstad and Omre, 2010; Ulvmoen and Omre, 2010). Some of these inversion methods are summarized in Doyen (2007) and Bosch et al. (2010).

In the previous literature, the distribution of the input rock properties is assumed from prior geologic information or estimated from actual measurements, then a training set of samples is generated using Monte Carlo simulations, and the set of predictions is obtained by applying the rock physics model (velocity-porosity, velocity-pressure, velocity-saturation, and so on) to the initial training data set. If the model is not linear, the initial distribution shape is not preserved and the posterior probability function has to be numerically estimated. In our work, we introduce the analytical solution of the problem so that the posterior probability function has a closed analytical form. For demonstration purposes, most of the examples are shown for the univariate case. The method is then extended to the multivariate case. The main advantage is the analytical solution of the uncertainty quantification problem. However, we warn the reader that for some rock physics models, the multivariate formulation can be complicated and Monte Carlo simulations can provide good approximations. On the other hand, if the output distribution is not Gaussian or more generally if it cannot be approximated by an analytical form, the probability density function (PDF) approximation from Monte Carlo samples can introduce a bias in the uncertainty quantification of the output PDF.

In this paper, we first present the general method and then show the application to a well-known rock physics model, Gassmann's equations (Mavko et al., 2009) with different uncertain input parameters, such as porosity and saturation, and different outputs, such as dry and saturated rock bulk moduli. The same method is then applied to other rock physics models, such as an empirical velocity prediction relation (Raymer's model), a theoretical granular media model (Dvorkin's soft-sand model), and an inclusion model (Kuster-Toksöz). In the last part, we show basic examples of applications to laboratory measurements and well-log data sets. In this formulation, we only consider uncertainty in the input data. Spatial uncertainty is not included. However, if an analytical solution of the uncertainty propagation problem is available, Markov chain Monte
Carlo methods can be used to sample from the output distributions and include spatial correlation.

\section{METHODOLOGY}

In this section, we present the mathematical theory to apply a rock physics model to a random variable, or a PDF, instead of the traditional approach where we apply the rock physics model to a deterministic value.

We first consider a variable $X$ whose value is uncertain, for example, porosity. In statistics, such a variable is called a random variable. A random variable (or stochastic variable) is a variable whose value is subject to variations and cannot be deterministically assessed. In other words, the specific value cannot be predicted with certainty before an experiment. All the rock properties in the subsurface can be considered as random variables because they cannot be measured exactly.

We denote with $f_{X}(x)$ the probability distribution of a continuous random variable $X$. A probability distribution is a function that gives the probability for each subset of values of the random variable. Mathematically, we define the PDF using the following equation:

$$
f_{X}(x) d x=P(x<X \leq x+d x),
$$

where $d x$ is a differential element of infinitesimal length and $P(x<$ $X \leq x+d x)$ is the probability of $X$ assuming a value between $x$ and $x+d x$. We recall that because a continuous variable can take any value on the real axis, there is an infinite number of possible realizations; therefore, the probability that a continuous variable $X$ will take the single value $x$ is 0 , i.e., $P(X=x)=0$. This is why we introduce the differential in the definition. Because occurrences in different intervals are mutually exclusive, we can compute the probability of the outcome of $X$ being in the interval $[a, b]$ using the integral form:

$$
P(a<X<b)=\int_{a}^{b} f_{X}(x) d x .
$$

A PDF must satisfy the following properties:

$$
\begin{array}{r}
0 \leq f_{X}(x) \leq+\infty \\
\int \quad \int f_{X}(x) d x=1 .
\end{array}
$$

These conditions guarantee that the probability values cannot be negative nor greater than 1 .

A continuous random variable is completely defined by its PDF; however, in some special cases, the complete PDF is not necessary and the distribution of the continuous random variable can be described by a finite number of parameters: These parameters are called the moments of random variables. Examples of moments are the mean and the variance.

The mean is the most common measure used to describe the most likely value that a random variable can get. The mean of a continuous random variable is defined as 


$$
\mu_{X}=\int x f_{X}(x) d x
$$

However, the mean cannot describe how uncertain the random variable is. A common measure for the uncertainty is the variance:

$$
\sigma_{X}^{2}=\int\left(x-\mu_{X}\right)^{2} f_{X}(x) d x
$$

The variance describes the spread of the distribution around the mean. The standard deviation is the square root of the variance.

Different PDFs can be used for continuous random variables. For most of them, if we know the shape of the distribution and the mean and the variance, the PDF is completely defined. The concepts of PDF, and as a consequence the concepts of mean and variance, can be extended to the multivariate domain, but for the mathematical formulation of the rock physics problem, we will limit our description to the univariate case.

The most common PDF is the Gaussian distribution. We say that a random variable $X$ is distributed according to a Gaussian distribution $N\left(\mu_{X}, \sigma_{X}^{2}\right)$ with mean $\mu_{X}$ and variance $\sigma_{X}^{2}$, if its PDF $f_{X}(x)$ can be written as

$$
f_{X}(x)=\frac{1}{\sqrt{2 \pi \sigma_{X}^{2}}} e^{-\frac{1\left(x-\mu_{X}\right)^{2}}{2} \sigma_{X}^{2}} .
$$

The Gaussian distribution is symmetric and can be used to describe many phenomena in nature.

Another commonly used distribution is the uniform distribution over a given interval, in which a random variable is equally likely to have any value in the assigned interval. In other words, the PDF is constant over the assigned range. The uniform distribution is sometimes called noninformative because it does not provide any additional knowledge other than the interval boundaries.

The problem that we face in this paper is to derive analytical expressions for nonlinear transformations of the distribution of a given random variable. If the transformation is linear, then the shape of the distribution is preserved. If the PDF is fully described by the mean and the variance, such as for the Gaussian distribution, to compute the PDF of the predicted variable we just have to compute the mean and the variance of the new distribution. For example, if $X$ is distributed according to a Gaussian distribution $N\left(\mu_{X}, \sigma_{X}^{2}\right)$ with mean $\mu_{X}$ and variance $\sigma_{X}^{2}$, and we apply a linear transformation $Y=g(X)=a X+b$, then $Y$ is still distributed according to a Gaussian distribution $N\left(a \mu_{X}+b, a^{2} \sigma_{X}^{2}\right)$ with mean

$$
\mu_{Y}=a \mu_{X}+b
$$

and variance

$$
\sigma_{Y}^{2}=a^{2} \sigma_{X}^{2}
$$

Similarly, if $X$ is distributed according to a uniform distribution over the interval $[c, d], X \sim U([c, d])$ and we apply a linear transformation $Y=g(X)=a X+b$, then $Y$ is still distributed according to a uniform distribution $Y \sim U([a c+b, a d+b])$.

This result is intuitive because if we take a linear transformation of a distribution, we can shift the mean and change the variance, i.e., the spread around the mean, but we cannot distort the shape of the distribution. For example, we can assume that porosity in a rock is distributed according to a Gaussian distribution (neglecting low probability values for porosities lower or higher than physical bounds). We then apply a rock physics model to compute the corresponding velocity distribution. If the rock physics model is linear, then the distribution of P-wave velocity is still Gaussian (Figure 1) and we can compute the mean and the variance using equations 8 and 9.

If the rock physics model is nonlinear, then the shape of the input distribution, for instance the Gaussian distribution of porosity, is not necessarily preserved in the distribution of the predicted variable, for instance, the distribution of P-wave velocity (Figure 2). To overcome this problem, a Monte Carlo simulation can be introduced to estimate the posterior distribution of the predicted variable, in our example, P-wave velocity. For an accurate estimation, the Monte Carlo simulation requires a training data set of a very large number a)

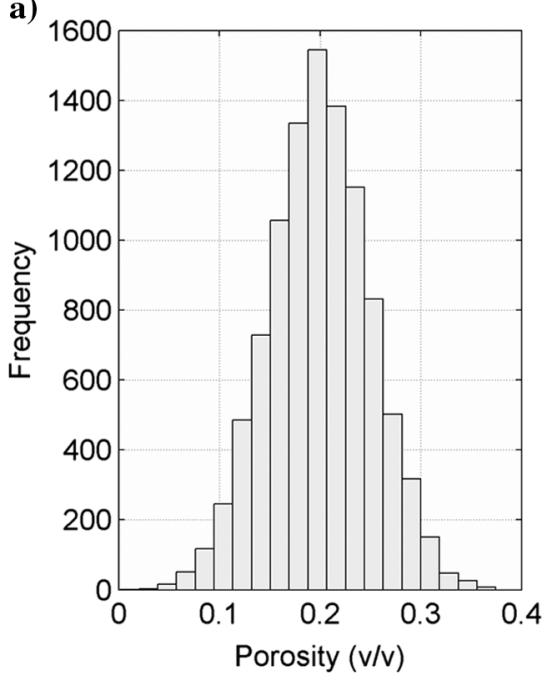

b)

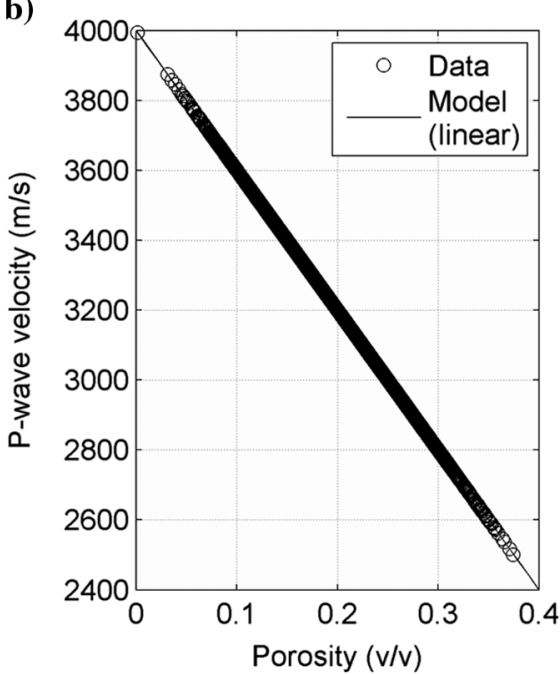

c)

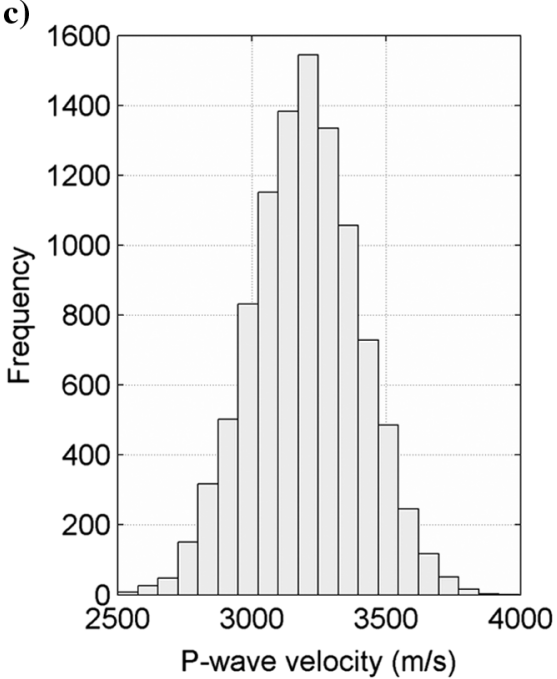

Figure 1. Linear rock physic model applied to a data set of 10,000 samples: (a) input porosity distribution, (b) linear rock physics model, (c) predicted P-wave velocity distribution. 
of samples. Furthermore, when the distribution of the Monte Carlo samples is not Gaussian, it is often necessary to approximate the distribution of the output samples with a parametric distribution, which can lead to errors in the uncertainty quantification problem. We propose here to exactly solve this problem using an analytical formulation. The analytical solution can be used in inverse problem applications, such as seismic reservoir characterization and reservoir modeling.

The method we propose is based on the following theorem (Papoulis, 1984): if $X$ is distributed according to a PDF $f_{X}(x)$, and $Y$ is equal to $Y=g(X)$, where $g$ is any transformation, linear or nonlinear, then the PDF $f_{Y}(y)$ can be computed as

$$
f_{Y}(y)=\sum_{i=1}^{n} \frac{f_{X}\left(x_{i}\right)}{\left|\frac{d}{d x} g\left(x_{i}\right)\right|},
$$

where $x_{i}$, for $i=1, \ldots, n$, are the real roots of the function $Y=g(X)$.

If $X$ is the uncertain input variable with a known PDF, $Y$ is the prediction, and $g$ is the rock physics model, then we can compute the PDF $f_{Y}(y)$ in three steps:

1) We first compute the uncertain input variable as a function of the prediction $X=g^{-1}(Y)$.

2) We compute the derivative of the rock physics model $\frac{d}{d X} g(X)$.

3) We apply equation 10 .

In a multidimensional problem, the workflow is similar and aims to determine the joint density $f_{Z, W}(z, w)$ of two random variables $(Z, W)$ that are functions of two other random variables $(X, Y)$

$$
\left\{\begin{array}{l}
Z=g(X, Y) \\
W=h(X, Y)
\end{array}\right.
$$

in terms of joint density of $(X, Y)$. It is important to consider the joint density of the random variables in order to account for the correlation between the variables. To find $f_{Z, W}(z, w)$, we first solve the system in equation 11 to find the real roots $\left(x_{i}, y_{i}\right)$ for $i=1, \ldots, n$. Then,

$$
f_{Z, W}(z, w)=\sum_{i=1}^{n} \frac{f_{X, Y}\left(x_{i}, y_{i}\right)}{\left|J\left(x_{i}, y_{i}\right)\right|},
$$

where $J\left(x_{i}, y_{i}\right)$ is the Jacobian of the transformation in equation 11 computed in the root $\left(x_{i}, y_{i}\right)$ and $\left|J\left(x_{i}, y_{i}\right)\right|$ is the determinant of the Jacobian (Papoulis, 1984). The extension to the multivariate case has been illustrated for the bivariate case, but the formulation is valid for any finite number of variables. When the analytical form of the rock physics model is too complicated to analytically compute equation 12, analytical approximations can be introduced using Talyor's series expansions and first order approximations.

In the following paragraphs, we present several applications and examples in the rock physics domain: Gassmann's fluid equation, Raymer's velocity prediction, Dvorkin's soft-sand model, and Kuster-Toksöz's inclusion model.

\section{Gassmann's equation with uncertain porosity}

One of the most popular rock physics model is Gassmann's equation for fluid substitution in porous rocks (Mavko et al., 2009). The idea of Gassmann's equation is to predict elastic moduli and velocities of rocks saturated with different fluids. For example, we could be interested in predicting velocities of gas-saturated rocks, but well logs only contain measurements for oil-saturated and/or water-saturated rocks.

Gassmann's theory (Mavko et al., 2009) assumes a homogeneous mineral modulus $\left(K_{\mathrm{mat}}\right)$ and statistical isotropy of the pore space, and it is valid at low frequencies where the induced pore pressure is equilibrated through the pore space. Gassmann's equation states that when a rock is loaded with a fluid under an increment of pore pressure, the shear modulus of the saturated rock $\left(\mu_{\text {sat }}\right)$ is not affected by the fluid change, and it is equal to the dry-rock shear modulus $\left(\mu_{\mathrm{sat}}=\mu_{\mathrm{dry}}\right)$, whereas the bulk modulus of the saturated rock $\left(K_{\text {sat }}\right)$ can be computed using the following equation:
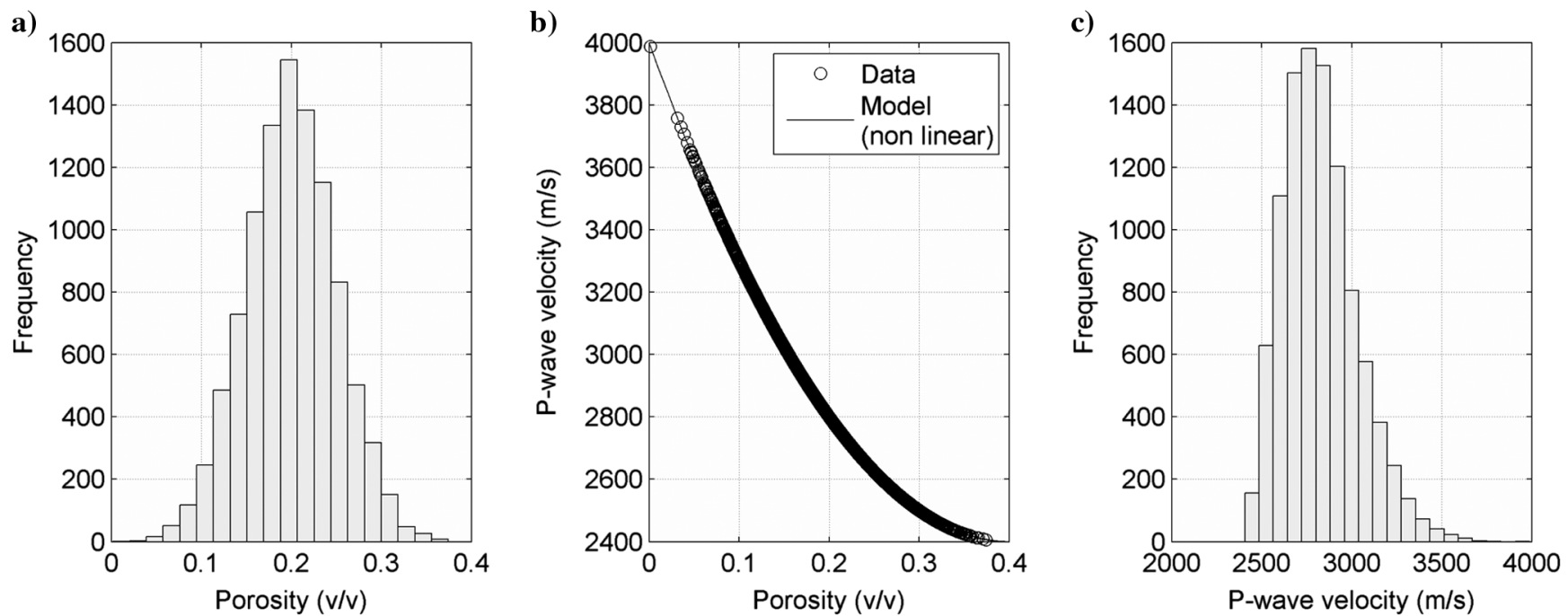

Figure 2. Nonlinear rock physic model applied to a data set of 10,000 samples: (a) input porosity distribution, (b) nonlinear rock physics model, (c) predicted P-wave velocity distribution. 


$$
K_{\mathrm{sat}}=K_{\mathrm{dry}}+\frac{\left(1-\frac{K_{\mathrm{dry}}}{K_{\mathrm{mat}}}\right)^{2}}{\frac{\phi}{K_{\mathrm{fl}}}+\frac{1-\phi}{K_{\mathrm{mat}}}-\frac{K_{\mathrm{dry}}}{K_{\mathrm{mat}}^{2}}}
$$

where $K_{\text {mat }}$ is the bulk modulus of the mineral frame, $K_{\mathrm{fl}}$ is the bulk modulus of the fluid, $K_{\text {dry }}$ is the dry rock bulk modulus, and $\phi$ is the porosity. Similarly, the dry-rock bulk modulus $K_{\text {dry }}$ can be derived from the saturated-rock bulk modulus using the inverse equation:

$$
K_{\mathrm{dry}}=\frac{K_{\mathrm{sat}}\left(\phi \frac{K_{\mathrm{mat}}}{K_{\mathrm{fl}}}+1-\phi\right)-K_{\mathrm{mat}}}{\phi \frac{K_{\mathrm{mat}}}{K_{\mathrm{fl}}}+\frac{K_{\mathrm{sat}}}{K_{\mathrm{mat}}}-1-\phi} .
$$

Gassmann's equations allow predicting saturated-rock moduli from dry-rock moduli and vice versa when the properties of the matrix mineralogical components, the properties of the fluids, and the porosity of the rock are known, but the most common application is predicting the saturated-rock moduli when one fluid is replaced with another.

These equations are generally applied to scalar values or vectors of porosity. In our application, we use a probability distribution for an uncertain variable, for example, porosity, to compute the saturated-rock bulk modulus using equation 10 . To apply equation 10 , we first compute the real roots of equation 13. If we assume that $K_{\text {mat }}, K_{\mathrm{fl}}$, and $K_{\text {dry }}$ are measured values, in other words, if $K_{\text {mat }}$, $K_{f l}$, and $K_{\text {dry }}$ are constant and $K_{\text {sat }}$ is only a function of $\phi$, then equation 13 has a single solution:

$$
\phi_{1}=\frac{K_{f l}\left(K_{\mathrm{mat}}-K_{\mathrm{dry}}\right)\left(K_{\mathrm{mat}}-K_{\mathrm{sat}}\right)}{K_{\mathrm{mat}}\left(K_{\mathrm{mat}}-K_{\mathrm{fl}}\right)\left(K_{\mathrm{sat}}-K_{\mathrm{dry}}\right)}
$$

for every value $K_{\text {sat }}$

The derivative of $K_{\text {sat }}$ with respect to $\phi$ is

$$
\begin{aligned}
\frac{d}{d \phi} K_{\mathrm{sat}}(\phi) & =-\frac{\left(\frac{1}{K_{\mathrm{fl}}}-\frac{1}{K_{\mathrm{mat}}}\right)\left(1-\frac{K_{\mathrm{dry}}}{K_{\mathrm{mat}}}\right)^{2}}{\left[\phi\left(\frac{1}{K_{\mathrm{fl}}}-\frac{1}{K_{\mathrm{mat}}}\right)+\frac{1}{K_{\mathrm{mat}}}\left(1-\frac{K_{\mathrm{dry}}}{K_{\mathrm{mat}}}\right)\right]^{2}} \\
& =\frac{K_{\mathrm{mat}} K_{\mathrm{fl}}\left(K_{\mathrm{dry}}-K_{\mathrm{mat}}\right)^{2}\left(K_{\mathrm{fl}}-K_{\mathrm{mat}}\right)}{\left[K_{\mathrm{dry}} K_{\mathrm{fl}}-K_{\mathrm{mat}}\left(K_{\mathrm{fl}}-\phi K_{\mathrm{fl}}+\phi K_{\mathrm{mat}}\right)\right]^{2}}
\end{aligned}
$$

(see Appendix A for the details of the derivation).

If we assume that porosity is distributed according to a Gaussian distribution $\phi \sim N\left(\mu_{\phi}, \sigma_{\phi}^{2}\right)$ with mean $\mu_{\phi}$ and variance $\sigma_{\phi}^{2}$, by applying equation 10 , and using linear algebra, we obtain that the distribution $f_{K_{\text {sat }}}\left(K_{\text {sat }}\right)$ of the saturated-rock bulk modulus is

$$
\begin{aligned}
f_{K_{\text {sat }}}\left(K_{\text {sat }}\right) & =\frac{f_{\phi}\left(\phi=\phi_{1}\right)}{\left|\frac{d}{d \phi} K_{\text {sat }}(\phi)\right|_{\mid \phi=\phi_{1}}} \\
& =\frac{K_{\mathrm{fl}}\left(K_{\mathrm{dry}}-K_{\text {mat }}\right)^{2}}{K_{\text {mat }}\left(K_{\text {mat }}-K_{\mathrm{fl}}\right)\left(K_{\text {dry }}-K_{\text {sat }}\right)^{2}} \frac{1}{\sqrt{2 \pi \sigma_{\phi}^{2}}} e^{-\frac{\left(\phi_{1}-\mu_{\phi}\right)^{2}}{2 \sigma_{\phi}^{2}}},
\end{aligned}
$$

where we remove the absolute value because $K_{\text {mat }}>K_{\mathrm{fl}}$. In most practical applications, $K_{\text {dry }}$ cannot be measured and it is computed using another rock physics model. For example, by using the concept of critical porosity $\phi_{0}$ in Nur's model (Mavko et al., 2009) we can write the dry-rock bulk modulus as a function of the matrix bulk modulus and the porosity:

$$
K_{\text {dry }}=K_{\text {mat }}\left(1-\frac{\phi}{\phi_{0}}\right) \text {. }
$$

Under this assumption, we can combine the rock physics model in equation 18 with Gassmann's equation in equation 13 to obtain the deterministic expression for the saturated-rock bulk modulus, which only depends on $K_{\mathrm{mat}}, K_{\mathrm{fl}}$, and $\phi$. Then, we can apply the same approach based on equation 10 , to derive the posterior distribution of $K_{\text {sat }}$ under the assumption that $K_{\text {mat }}$ and $K_{\mathrm{fl}}$ are constant. Skipping the calculations, we obtain

$$
\begin{aligned}
f_{K_{\mathrm{sat}}}\left(K_{\mathrm{sat}}\right) & =\frac{f_{\phi}\left(\phi=\phi_{1}\right)}{\left|\frac{d}{d \phi} K_{\mathrm{sat}}(\phi)\right|_{\mid \phi=\phi_{1}}} \\
& =\left|\frac{K_{\mathrm{fl}}-\phi_{0}\left(K_{\mathrm{mat}}-K_{\mathrm{fl}}\right)}{K_{\mathrm{mat}}\left(K_{\mathrm{fl}}-K_{\mathrm{mat}}\right)}\right| \frac{1}{\sqrt{2 \pi \sigma_{\phi}^{2}}} e^{-\frac{\left(\phi_{1}-\mu_{\phi}\right)^{2}}{2 \sigma_{\phi}^{2}}},
\end{aligned}
$$

where

$$
\phi_{1}=\frac{\left(\phi_{0}\left(K_{\mathrm{fl}}-K_{\mathrm{mat}}\right)-K_{\mathrm{fl}}\right)\left(K_{\mathrm{mat}}-K_{\mathrm{sat}}\right)}{K_{\mathrm{mat}}\left(K_{\mathrm{fl}}-K_{\mathrm{mat}}\right)} .
$$

In the second example, we use the same approach to derive the distribution of the dry-rock bulk modulus $f_{K_{\text {dry }}}\left(K_{\text {dry }}\right)$ when $K_{\text {mat }}$, $K_{\mathrm{fl}}$, and $K_{\text {sat }}$ are known measured values and $\phi \sim N\left(\mu_{\phi}, \sigma_{\phi}^{2}\right)$. If we assume that $K_{\text {mat }}, K_{\mathrm{fl}}$, and $K_{\text {sat }}$ are constant, in other words, if $K_{\mathrm{dry}}$ is only a function of $\phi$, then equation 14 has only a single solution:

$$
\phi_{1}=\frac{K_{\mathrm{fl}}\left(K_{\mathrm{mat}}-K_{\mathrm{dry}}\right)\left(K_{\mathrm{mat}}-K_{\mathrm{sat}}\right)}{K_{\mathrm{mat}}\left(K_{\mathrm{mat}}-K_{\mathrm{fl}}\right)\left(K_{\mathrm{sat}}-K_{\mathrm{dry}}\right)}
$$

for every value $K_{\mathrm{dry}}$. The derivative of $K_{\mathrm{dry}}$ with respect to $\phi$ is

$$
\frac{d}{d \phi} K_{\mathrm{dry}}(\phi)=\frac{K_{\mathrm{mat}} K_{\mathrm{fl}}\left(K_{\mathrm{mat}}-K_{\mathrm{fl}}\right)\left(K_{\mathrm{sat}}-K_{\mathrm{mat}}\right)^{2}}{\left(\phi K_{\mathrm{mat}}^{2}-\phi K_{\mathrm{mat}} K_{\mathrm{fl}}+K_{\mathrm{sat}} K_{\mathrm{fl}}-K_{\mathrm{mat}} K_{\mathrm{fl}}\right)} .
$$

The distribution of the dry-rock bulk modulus $f_{K_{\mathrm{dry}}}\left(K_{\mathrm{dry}}\right)$ when $\phi \sim$ $N\left(\mu_{\phi}, \sigma_{\phi}^{2}\right)$ is then

$$
\begin{aligned}
f_{K_{\mathrm{dry}}}\left(K_{\mathrm{dry}}\right) & =\frac{f_{\phi}\left(\phi=\phi_{1}\right)}{\left|\frac{d}{d \phi} K_{\mathrm{dry}}(\phi)\right|_{\mid \phi=\phi_{1}}} \\
& =\frac{K_{\mathrm{fl}}\left(K_{\mathrm{mat}}-K_{\mathrm{sat}}\right)^{2}}{K_{\text {mat }}\left(K_{\mathrm{mat}}-K_{\mathrm{fl}}\right)\left(K_{\mathrm{dry}}-K_{\mathrm{sat}}\right)^{2}} \frac{1}{\sqrt{2 \pi \sigma_{\phi}^{2}}} e^{-\frac{\left(\phi_{1}-\mu_{\phi}\right)^{2}}{2 \sigma_{\phi}^{2}}},
\end{aligned}
$$

where we removed the absolute value because $K_{\text {mat }}>K_{\mathrm{fl}}$. 


\section{Gassmann's equation with uncertain saturation}

Similarly, we can apply equation 10 to derive the analytical formulation of the saturated-rock bulk modulus when water saturation $s_{w}$ is described by a probability distribution rather than a definite value. As we can observe in equation $13, s_{w}$ does not explicitly appear in Gassmann's equation, but the changes in water saturation affect the fluid bulk modulus $K_{\mathrm{fl}}$.

Laboratory experiments show that not only the fluid saturations but also the spatial distribution of the fluids inside the pore space (Mavko et al., 2009) affect velocity values. Different equations can be used to describe the different spatial distributions: For patchy saturation, we can use a linear average of the fluid bulk moduli, whereas for a homogeneous fluid distribution, the harmonic average of the fluid moduli is more realistic. In this section, we analyze both cases.

If we assume a patchy saturation of two fluid phases, for example, water and gas, the fluid mixing law to compute the fluid bulk modulus can be written as a linear average:

$$
K_{\mathrm{fl}}=K_{w} s_{w}+K_{g}\left(1-s_{w}\right)
$$

where $K_{w}$ is the bulk modulus of water and $K_{g}$ is the bulk modulus of gas.

We then assume that $K_{\text {mat }}, K_{\text {dry }}$, and $\phi$ are constant (and not equal 0 ), and we specify a probability distribution $f_{S_{w}}\left(s_{w}\right)$ for water saturation. Under these assumptions, the inverse function can be written as

$$
s_{w_{1}}=\frac{K_{\mathrm{dry}}\left(-\phi K_{\mathrm{mat}}^{2}+\xi K_{g}\right)+K_{\mathrm{mat}}\left(\phi K_{\mathrm{mat}} K_{\mathrm{sat}}-\psi K_{g}\right)}{\left(K_{g}-K_{w}\right)\left(\xi K_{\mathrm{dry}}-\psi K_{\mathrm{mat}}\right)},
$$

where $\xi=K_{\text {mat }}+\phi K_{\text {mat }}-K_{\text {sat }}$ and $\psi=K_{\text {mat }}+\phi K_{\text {sat }}-K_{\text {sat }}$ and the derivative of $K_{\text {sat }}$ with respect to $s_{w}$ is

$$
\begin{aligned}
& \frac{d}{d s_{w}} K_{\text {sat }}\left(s_{w}\right) \\
& =\frac{\phi\left(K_{w}-K_{g}\right)\left(1-\frac{K_{\mathrm{dry}}}{K_{\text {mat }}}\right)^{2}}{\left(K_{w} s_{w}+K_{g}\left(1-s_{w}\right)\right)^{2}\left[\frac{1}{K_{\text {mat }}}\left(1-\frac{K_{\text {dry }}}{K_{\text {mat }}}\right)+\phi\left(\frac{1}{K_{w} s_{w}+K_{g}\left(1-s_{w}\right)}-\frac{1}{K_{\text {mat }}}\right)\right]^{2}} .
\end{aligned}
$$

By applying equation 10, we obtain that the distribution $f_{K_{\text {sat }}}\left(K_{\text {sat }}\right)$ of the saturated-rock bulk modulus is

$$
\begin{aligned}
f_{K_{\text {sat }}}\left(K_{\text {sat }}\right) & =\frac{f_{s_{w}}\left(s_{w}=s_{w_{1}}\right)}{\left|\frac{d}{d s_{w}} K_{\text {sat }}\left(s_{w}\right)\right|_{\mid s_{w}=s_{w_{1}}}} \\
& =\frac{\phi K_{\text {mat }}^{2}\left(K_{\mathrm{dry}}-K_{\text {mat }}\right)^{2}}{\left(K_{w}-K_{g}\right)\left(\xi K_{\text {dry }}-\psi K_{\text {mat }}\right)^{2}} f_{s_{w}}\left(s_{w}=s_{w_{1}}\right),
\end{aligned}
$$

where $f_{s_{w}}\left(s_{w}=s_{w_{1}}\right)$ is the input PDF of water saturation evaluated in $s_{w_{1}}$ and where we removed the absolute value because $K_{w}>K_{g}$.

If we assume a homogeneous saturation of two fluid phases, water and gas, the fluid mixing law to compute the fluid bulk modulus, can be written as a harmonic average:

$$
K_{\mathrm{fl}}=\frac{1}{\frac{s w}{K_{w}}+\frac{(1-s w)}{K_{g}}}
$$

and with similar computations, we can derive the distribution $f_{K_{\text {sat }}}\left(K_{\text {sat }}\right)$ of the saturated-rock bulk modulus

$$
\begin{aligned}
f_{K_{\mathrm{sat}}}\left(K_{\mathrm{sat}}\right) & =\frac{f_{s_{w}}\left(s_{w}=s_{w_{1}}\right)}{\left|\frac{d}{d s_{w}} K_{\mathrm{sat}}\left(s_{w}\right)\right|_{\mid s_{w}=s_{w_{1}}}} \\
& =\frac{K_{w} K_{g}\left(K_{\mathrm{dry}}-K_{\mathrm{mat}}\right)^{2}}{\phi K_{\mathrm{mat}}^{2}\left(K_{w}-K_{g}\right)\left(K_{\mathrm{dry}}-K_{\mathrm{sat}}\right)^{2}} f_{s_{w}}\left(s_{w}=s_{w_{1}}\right),
\end{aligned}
$$

where $K_{w}>K_{g}$ and

$$
s_{w_{1}}=\frac{K_{w}\left[K_{\mathrm{dry}}\left(-\phi K_{\mathrm{mat}}^{2}+\xi K_{g}\right)+K_{\mathrm{mat}}\left(\phi K_{\mathrm{mat}} K_{\mathrm{sat}}-\psi K_{g}\right)\right]}{\phi K_{\mathrm{mat}}^{2}\left(K_{g}-K_{w}\right)\left(K_{\mathrm{dry}}-K_{\mathrm{sat}}\right)} .
$$

Another common mixing law for fluid mixtures is given by Brie's equation (Brie et al., 1995). The Voigt average (used for patchy saturation, equation 24) and the Reuss average (used for homogeneous saturation, equation 28) represent the upper and lower bounds for a mixture of fluids; however, in most of the cases, data fall in between these two bounds and Brie's equation provides a better fit for these data sets thanks to the exponent that can be calibrated to the data (Brie et al., 1995).

It is important to point out that generally, water saturation is not distributed according to a Gaussian distribution: Especially when the fluid distribution is not patchy, it is more likely to observe values close to the extremes of the physical range, $0 \%$ and $100 \%$. This behavior can be better described by a beta distribution (Papoulis, 1984). The PDF of a random variable $X$ distributed according to a beta distribution with parameters $\alpha$ and $\beta$ can be written as

$$
f_{X}(x)=\frac{x^{\alpha-1}(1-x)^{\beta-1}}{B(\alpha, \beta)}
$$

where $\alpha$ and $\beta$ are two parameters that control the shape of the distribution and are related to the mean and the variance and $B($.$) is the$ beta function (Papoulis, 1984). If we assume that $s_{w} \sim B(\alpha, \beta)$ and we assume a homogeneous fluid distribution, then the distribution $f_{K_{\text {sat }}}\left(K_{\text {sat }}\right)$ of the saturated-rock bulk modulus becomes

$$
\begin{aligned}
f_{K_{\mathrm{sat}}}\left(K_{\mathrm{sat}}\right) & =\frac{f_{\phi}\left(s_{w}=s_{w_{1}}\right)}{\left|\frac{d}{d s_{w}} K_{\mathrm{sat}}\left(s_{w}\right)\right|_{\mid s_{w}=s_{w_{1}}}} \\
& =\frac{K_{w} K_{g}\left(K_{\mathrm{dry}}-K_{\mathrm{mat}}\right)^{2}}{\phi K_{\mathrm{mat}}^{2}\left(K_{w}-K_{g}\right)\left(K_{\mathrm{dry}}-K_{\mathrm{sat}}\right)^{2}} \frac{s_{w_{1}}^{\alpha-1}\left(1-s_{w_{1}}\right)^{\beta-1}}{B(\alpha, \beta)},
\end{aligned}
$$

and $s_{w_{1}}$ is the same as in equation 30 .

All these equations can be applied to Gassmann-Mavko equation (Mavko et al., 1995) in which the bulk modulus is replaced by the 
compressional modulus $M_{\text {sat }}=K_{\text {sat }}+4 / 3 \mu_{\text {sat }}$. The GassmannMavko equation is often used to perform fluid substitution when $\mathrm{S}$-wave velocities are not available. The equation predicts the compressional modulus of a saturated rock when the initial fluid is replaced with another. The use of the compressional modulus instead of the bulk modulus allows performing fluid substitution without the explicit knowledge of the shear modulus, hence the value of the S-wave velocity (Mavko et al., 1995).

\section{Raymer's model with uncertain porosity}

Another very common equation used in rock physics is Raymer's equation (Raymer et al., 1980). Raymer's equation is an empirical equation to predict $\mathrm{P}$-wave velocity $\left(V_{\mathrm{P}}\right)$ given the velocity of the compressional waves in the solid $\left(V_{\mathrm{P}}^{\mathrm{mat}}\right)$ and in the fluid $\left(V_{\mathrm{P}}^{\mathrm{fl}}\right)$ and the porosity of the rock. The equation has different formulations for different porosity ranges; in this work, for simplicity we only focus on the porosity interval $[0,0.37]$, where the equation for $V_{\mathrm{P}}$ can be written as

$$
V_{\mathrm{P}}=(1-\phi)^{2} V_{\mathrm{P}}^{\mathrm{mat}}+\phi V_{\mathrm{P}}^{\mathrm{fl}}
$$

Similarly to the previous examples, we first solve the equation for $\phi$ and obtain two solutions:

$$
\phi_{1,2}=\frac{-V_{\mathrm{P}}^{\mathrm{fl}}+2 V_{\mathrm{P}}^{\mathrm{mat}} \pm \sqrt{\left(V_{\mathrm{P}}^{\mathrm{fl}}\right)^{2}+4 V_{\mathrm{P}} V_{\mathrm{P}}^{\mathrm{mat}}-4 V_{\mathrm{P}}^{\mathrm{fl}} V_{\mathrm{P}}^{\mathrm{mat}}}}{2 V_{\mathrm{P}}^{\mathrm{mat}}},
$$

but we only use $\phi_{1}$ because $\phi_{2}$ provides values greater than 1, and we compute the derivative of $V_{\mathrm{P}}$ with respect to $\phi$, assuming that $V_{\mathrm{P}}^{\mathrm{mat}}$ and $V_{\mathrm{P}}^{\mathrm{fl}}$ are known constant values:

$$
\frac{d}{d \phi} V_{\mathrm{P}}(\phi)=V_{\mathrm{P}}^{\mathrm{fl}}-2(1-\phi) V_{\mathrm{P}}^{\mathrm{mat}}
$$

and by applying equation 10 and using linear algebra, we obtain

$$
f_{V_{\mathrm{P}}}\left(V_{\mathrm{P}}\right)=\frac{1}{\sqrt{\left(V_{\mathrm{P}}^{\mathrm{fl}}\right)^{2}+4 V_{\mathrm{P}} V_{\mathrm{P}}^{\mathrm{mat}}-4 V_{\mathrm{P}}^{\mathrm{fl}} V_{\mathrm{P}}^{\mathrm{mat}}}} f_{\phi}\left(\phi=\phi_{1}\right) .
$$

Dvorkin (2008) extends Raymer's equation to $V_{\mathrm{S}}$ predictions:

$$
V_{\mathrm{S}}=(1-\phi)^{2} V_{\mathrm{S}}^{\mathrm{mat}} \sqrt{\frac{(1-\phi) \rho^{\mathrm{mat}}}{(1-\phi) \rho^{\mathrm{mat}}+\phi \rho^{\mathrm{fl}}}},
$$

where $\rho^{\text {mat }}$ is the density of the matrix and $\rho^{\mathrm{fl}}$ is the density of the fluid. Then, the corresponding PDF for $V_{\mathrm{S}}$ can be derived in the same way as in equation 36 .

\section{Soft-sand model with uncertain porosity}

Raymer's equation is an empirical equation; however, several theoretical models have been developed to compute elastic moduli of porous materials: granular media models, inclusion models, etc. (see Mavko et al., 2009). In this section, we propagate the uncertainty of porosity to dry-rock bulk modulus predictions through the soft-sand model (Dvorkin and Nur, 1996). The soft-sand model belongs to the group of granular media models and is based on the Hertz-Mindlin contact theory. The soft-sand model extrapolates elastic property values in the porosity range between zero and the critical porosity by using a modified Hashin-Shtrikman lower bound (Hashin and Shtrikman, 1963; Mavko et al., 2009).

For porosity values ranging between zero and the critical porosity $\phi_{0}$, the soft-sand rock physics model connects the matrix elastic moduli $K_{\text {mat }}$ and $\mu_{\text {mat }}$ (elastic moduli at zero porosity) with the Hertz-Mindlin elastic moduli $K_{\mathrm{HM}}$ and $\mu_{\mathrm{HM}}$ (elastic moduli of the dry rock at critical porosity) using the modified HashinShtrikman lower bounds:

$$
\begin{gathered}
K_{\mathrm{dry}}=\left(\frac{\frac{\phi}{\phi_{0}}}{K_{\mathrm{HM}}+\gamma}+\frac{1-\frac{\phi}{\phi_{0}}}{K_{\mathrm{mat}}+\gamma}\right)^{-1}-\gamma, \\
\mu_{\mathrm{dry}}=\left(\frac{\frac{\phi}{\phi_{0}}}{K_{\mathrm{HM}}+\eta}+\frac{1-\frac{\phi}{\phi_{0}}}{\mu_{\mathrm{mat}}+\eta}\right)^{-1}-\eta,
\end{gathered}
$$

where

$$
\begin{aligned}
\gamma & =\frac{4}{3} \mu_{\mathrm{HM}}, \\
\eta & =\frac{1}{6 \mu_{\mathrm{HM}}} \frac{9 K_{\mathrm{HM}}+8 \mu_{\mathrm{HM}}}{K_{\mathrm{HM}}+2 \mu_{\mathrm{HM}}}, \\
K_{\mathrm{HM}} & =\sqrt[3]{\frac{P\left[n\left(1-\phi_{0}\right) \mu_{\mathrm{mat}}\right]^{2}}{18[\pi(1-\nu)]^{2}}} \\
\mu_{\mathrm{HM}} & =\frac{5-4 \nu}{5(2-\nu)} \sqrt[3]{\frac{3 P\left[n\left(1-\phi_{0}\right) \mu_{\mathrm{mat}}\right]^{2}}{2[\pi(1-\nu)]^{2}}},
\end{aligned}
$$

and where $\nu$ is the matrix Poisson's ratio, $n$ is the coordination number (average number of contacts per grain), and $P$ is the effective pressure (Mavko et al., 2009). In the following, we assume that all the parameters in equation 40 are constant. Under these assumptions, the dry-rock bulk modulus in equation 38 and the dry-rock shear modulus in equation 39 are only functions of porosity. By applying the proposed method, we first explicitly write porosity as a function of the dry-rock elastic moduli:

$$
\begin{aligned}
\phi_{1}^{K} & =\frac{\phi_{0}\left(K_{\mathrm{dry}}-K_{\mathrm{mat}}\right)\left(K_{\mathrm{HM}}+\gamma\right)}{\left(K_{\mathrm{HM}}+K_{\mathrm{mat}}\right)\left(K_{\mathrm{dry}}+\gamma\right)}, \\
\phi_{1}^{\mu} & =\frac{\phi_{0}\left(\mu_{\mathrm{dry}}-\mu_{\mathrm{mat}}\right)\left(\mu_{\mathrm{HM}}+\eta\right)}{\left(\mu_{\mathrm{HM}}+\mu_{\mathrm{mat}}\right)\left(\mu_{\mathrm{dry}}+\eta\right)},
\end{aligned}
$$

and then we compute the derivatives of equations 38 and 39:

$$
\begin{aligned}
& \frac{d}{d \phi} K_{\mathrm{dry}}(\phi)=\frac{\phi_{0}\left(K_{\mathrm{HM}}-K_{\mathrm{mat}}\right)\left(K_{\mathrm{HM}}+\gamma\right)\left(K_{\mathrm{mat}}+\gamma\right)}{\left(\phi K_{\mathrm{mat}}+K_{\mathrm{HM}}\left(\phi_{0}-\phi\right)+\phi_{0} \gamma\right)^{2}}, \\
& \frac{d}{d \phi} \mu_{\mathrm{dry}}(\phi)=\frac{\phi_{0}\left(\mu_{\mathrm{HM}}-\mu_{\mathrm{mat}}\right)\left(\mu_{\mathrm{HM}}+\eta\right)\left(\mu_{\mathrm{mat}}+\eta\right)}{\left(\phi \mu_{\mathrm{mat}}+\mu_{\mathrm{HM}}\left(\phi_{0}-\phi\right)+\phi_{0} \eta\right)^{2}},
\end{aligned}
$$


and finally we obtain the PDFs of $K_{\mathrm{dry}}$ and $\mu_{\mathrm{dry}}$ by applying equation 10:

$$
\begin{aligned}
& f_{K_{\text {dry }}}\left(K_{\text {dry }}\right)=\left|\frac{\phi_{0}\left(K_{\mathrm{HM}}+\gamma\right)\left(K_{\text {mat }}+\gamma\right)}{\left(K_{\mathrm{HM}}-K_{\text {mat }}\right)\left(K_{\text {dry }}+\gamma\right)^{2}}\right| f_{\phi}\left(\phi=\phi_{1}\right), \\
& f_{\mu_{\text {dry }}}\left(\mu_{\text {dry }}\right)=\left|\frac{\phi_{0}\left(\mu_{\mathrm{HM}}+\eta\right)\left(\mu_{\mathrm{mat}}+\eta\right)}{\left(\mu_{\mathrm{HM}}-\mu_{\mathrm{mat}}\right)\left(\mu_{\mathrm{dry}}+\eta\right)^{2}}\right| f_{\phi}\left(\phi=\phi_{1}\right) \text {. }
\end{aligned}
$$

The same analysis could be performed with the stiff-sand model (Gal et al., 1998), that uses a modified Hashin-Shtrikman upper bound, resulting in an increase of elastic property values compared to the soft-sand model. The calculations are similar to the softsand case.

\section{Inclusion model with uncertain porosity}

In this section, we repeat the same exercise presented in the previous section using an inclusion model. In inclusion models, the saturated rock is modeled as an elastic medium with holes (inclusions) within it. Several models have been proposed. In this example, we use one of the first formulations proposed by Kuster and Toksöz (1974), based on long-wavelength first-order scattering theory. The bulk and shear moduli, $K_{\mathrm{KT}}^{*}$ and $\mu_{\mathrm{KT}}^{*}$, of the effective medium are given by

$$
\begin{aligned}
& \left(K_{\mathrm{KT}}^{*}-K_{\mathrm{mat}}\right) \frac{K_{\mathrm{mat}}+\frac{4}{3} \mu_{\mathrm{mat}}}{K_{\mathrm{KT}}^{*}+\frac{4}{3} \mu_{\mathrm{mat}}}=\sum_{i=1}^{N} f_{i}\left(K_{\mathrm{KT}}^{*}-K_{\mathrm{mat}}\right) P_{i}, \\
& \left(\mu_{\mathrm{KT}}^{*}-\mu_{\mathrm{mat}}\right) \frac{\mu_{\mathrm{mat}}+\frac{1}{6} \mu_{\mathrm{mat}} \xi}{\mu_{\mathrm{KT}}^{*}+\frac{1}{6} \mu_{\mathrm{mat}} \xi}=\sum_{i=1}^{N} f_{i}\left(\mu_{\mathrm{KT}}^{*}-\mu_{\mathrm{mat}}\right) Q_{i},
\end{aligned}
$$

where $\xi=\left(9 K_{\text {mat }}+8 \mu_{\text {mat }}\right) /\left(K_{\text {mat }}+2 \mu_{\text {mat }}\right), N$ is the number of inclusions, $f_{i}$ is the fraction volume of the $i$ th inclusion, and $P_{i}$ and $Q_{i}$ are coefficients that depend on the geometry of the inclusion. Kuster and Toksöz (1974) propose the analytical formulation for different inclusion shapes, such as spheres, needles, disks, and penny cracks. For illustration purposes, in this example we assume spherical shapes; however, the mathematical formulation of the probabilistic rock physics approach does not depend on these coefficients because they are constant with respect to the volumetric fractions.

We assume for simplicity a single inclusion $f_{1}=\phi$ (for example, porosity filled by a single fluid phase), and we first derive the explicit expression of $K_{\mathrm{KT}}^{*}$ and $\mu_{\mathrm{KT}}^{*}$ :

$$
\begin{aligned}
K_{\mathrm{KT}}^{*} & =\frac{K_{\mathrm{mat}}\left(K_{\mathrm{mat}}+\frac{4}{3} \mu_{\mathrm{mat}}\right)+\frac{4}{3} \phi P_{1}\left(K_{1}-K_{\mathrm{mat}}\right) \mu_{\mathrm{mat}}}{\phi P_{1}\left(K_{\mathrm{mat}}-K_{1}\right)\left(K_{\mathrm{mat}}+\frac{4}{3} \mu_{\mathrm{mat}}\right)}, \\
\mu_{\mathrm{KT}}^{*} & =\frac{\mu_{\mathrm{mat}}\left(\mu_{\mathrm{mat}}+\frac{1}{6} \mu_{\mathrm{mat}} \xi\right)+\frac{1}{6} \phi \xi Q_{1}\left(\mu_{1}-\mu_{\mathrm{mat}}\right) \mu_{\mathrm{mat}}}{\phi Q_{1}\left(\mu_{\mathrm{mat}}-\mu_{1}\right)+\left(\mu_{\mathrm{mat}}+\frac{1}{6} \mu_{\mathrm{mat}} \xi\right)},
\end{aligned}
$$

where $K_{1}$ and $\mu_{1}$ are the elastic moduli of the component of the inclusion (for example, of the fluid) and $P_{1}$ and $Q_{1}$ are the coefficients that describe the shape of the pore space. For spherical pores
$P_{1}=\left(K_{\text {mat }}+4 / 3 \mu_{\text {mat }}\right) /\left(K_{1}+4 / 3 \mu_{\text {mat }}\right)$ and $Q_{1}=\left(\mu_{\text {mat }}+1 / 6 \mu_{\text {mat }} \xi\right) /$ $\left(\mu_{1}+1 / 6 \mu_{\text {mat }} \xi\right)$.

Similarly to what we have done for the previous examples, we compute the derivatives of the functions with respect to porosity and we evaluate them in the zero of the functions. The derivatives are

$$
\begin{aligned}
\frac{d}{d \phi} K_{\mathrm{KT}}^{*}(\phi) & =\frac{P_{1}\left(K_{1}-K_{\mathrm{mat}}\right)\left(K_{\mathrm{mat}}+\frac{4}{3} \mu_{\mathrm{mat}}\right)^{2}}{\left(\phi P_{1}\left(K_{\mathrm{mat}}-K_{1}\right)+\left(K_{\mathrm{mat}}+\frac{4}{3} \mu_{\mathrm{mat}}\right)\right)^{2}}, \\
\frac{d}{d \phi} \mu_{\mathrm{KT}}^{*}(\phi) & =\frac{\xi Q_{1}\left(\mu_{1}-\mu_{\mathrm{mat}}\right)\left(\mu_{\mathrm{mat}}+\frac{1}{6} \mu_{\mathrm{mat}} \xi\right)^{2}}{\left(\phi Q_{1}\left(\mu_{\mathrm{mat}}-\mu_{1}\right)+\left(\mu_{\mathrm{mat}}+\frac{1}{6} \mu_{\mathrm{mat}} \xi\right)\right)^{2}},
\end{aligned}
$$

and the zeros of the functions are

$$
\begin{gathered}
\phi_{1}^{K}=\frac{\left(K_{\mathrm{KT}}^{*}-K_{\mathrm{mat}}\right)\left(K_{\mathrm{mat}}+\frac{4}{3} \mu_{\mathrm{mat}}\right)}{P_{1}\left(K_{1}-K_{\mathrm{mat}}\right)+\left(K_{\mathrm{KT}}^{*}+\frac{4}{3} \mu_{\mathrm{mat}}\right)}, \\
\phi_{1}^{\mu}=\frac{\left(\mu_{\mathrm{KT}}^{*}-\mu_{\mathrm{mat}}\right)\left(\mu_{\mathrm{mat}}+\frac{1}{6} \mu_{\mathrm{mat}} \xi\right)}{Q_{1}\left(\mu_{1}-\mu_{\mathrm{mat}}\right)+\left(\mu_{\mathrm{KT}}^{*}+\frac{1}{6} \mu_{\mathrm{mat}} \xi\right)} .
\end{gathered}
$$

Finally,

$$
\begin{aligned}
f_{K_{\mathrm{KT}}^{*}}\left(K_{\mathrm{KT}}^{*}\right) & =\frac{\left(K_{\mathrm{mat}}+\frac{4}{3} \mu_{\mathrm{mat}}\right)^{2}}{P_{1}\left(K_{\mathrm{mat}}-K_{1}\right)\left(K_{\mathrm{KT}}^{*}+\frac{4}{3} \mu_{\mathrm{mat}}\right)^{2}} f_{\phi}\left(\phi_{1}^{K}\right), \\
f_{\mu_{\mathrm{KT}}^{*}}\left(\mu_{\mathrm{KT}}^{*}\right) & =\frac{\left(\mu_{\mathrm{mat}}+\frac{1}{6} \xi \mu_{\mathrm{mat}}\right)^{2}}{Q_{1}\left(\mu_{\mathrm{mat}}-\mu_{1}\right)\left(\mu_{\mathrm{KT}}^{*}+\frac{1}{6} \xi \mu_{\mathrm{mat}}\right)^{2}} f_{\phi}\left(\phi_{1}^{\mu}\right),
\end{aligned}
$$

where $K_{\text {mat }}>K_{1}$ and $\mu_{\text {mat }}>\mu_{1}$.

\section{Application in multivariate domains}

As described in the section "Methodology," the method can be applied to multiple functions of multiple random variables (equations 11 and 12). The mathematical formulation is similar to the univariate case, but because it requires the calculation of the determinant of the Jacobian of the physical model, the derivation is generally more complicated. For clarity of illustration, we show an example with a simple model (Nur et al., 1995) based on the concept of critical porosity. We chose this model for its simple analytical formulation; however, the method could be applied to any other model.

In this example, we assume a rock with two mineralogical components, quartz and clay. We assume to have two random variables, porosity $\phi$ and clay volume $c$. Nur's model provides an estimation of the bulk and shear moduli of the dry rock as follows:

$$
K_{\mathrm{dry}}=K_{\mathrm{mat}}\left(1-\frac{\phi}{\phi_{0}}\right), \quad \mu_{\mathrm{dry}}=\mu_{\mathrm{mat}}\left(1-\frac{\phi}{\phi_{0}}\right),
$$


where the elastic moduli of the matrix clearly depend on the clay volume $c$. For the elastic moduli expression, we choose the Voigt average:

$$
K_{\mathrm{mat}}=c K_{c}+(1-c) K_{q}, \quad \mu_{\mathrm{mat}}=c \mu_{c}+(1-c) \mu_{q} .
$$

Different averages could be used for the matrix: Reuss, Voigt, or Hill. The Voigt average was chosen for clarity of illustration. We leave the computation of the derivatives to the reader, and we directly show the Jacobian matrix:

$$
J=\left[\begin{array}{cc}
-\frac{c K_{c}+(1-c) K_{q}}{\phi_{0}} & \left(K_{c}-K_{q}\right)\left(1-\frac{\phi}{\phi_{0}}\right) \\
-\frac{c \mu_{c}+(1-c) \mu_{q}}{\phi_{0}} & \left(\mu_{c}-\mu_{q}\right)\left(1-\frac{\phi}{\phi_{0}}\right)
\end{array}\right],
$$

and the determinant is

$$
|J|=\frac{K_{q} \mu_{c} \phi}{\phi_{0}^{2}}-\frac{K_{c} \mu_{q} \phi}{\phi_{0}^{2}}-\frac{K_{q} \mu_{c}}{\phi_{0}}+\frac{K_{c} \mu_{q}}{\phi_{0}} .
$$

We finally compute the real roots of the system:

$$
\begin{aligned}
& \bar{\phi}=\frac{K_{\mathrm{dry}}\left(\mu_{q}-\mu_{c}\right)+K_{q}\left(\mu_{c}-\mu_{\mathrm{dry}}\right)-K_{c}\left(\mu_{q}-\mu_{\mathrm{dry}}\right)}{K_{q} \mu_{c}-K_{c} \mu_{q}} \phi_{0} \\
& \bar{c}=\frac{K_{\mathrm{dry}} \mu_{q}-\mu_{\mathrm{dry}} K_{q}}{\mu_{\mathrm{dry}}\left(K_{c}-K_{q}\right)-K_{\mathrm{dry}}\left(\mu_{c}-\mu_{q}\right)}
\end{aligned}
$$

For simplicity, we skip the algebraic computation and show the final PDF of $\left(K_{\text {dry }}, \mu_{\text {dry }}\right)$ :

$$
\begin{aligned}
& f_{K_{\mathrm{dry}}, \mu_{\mathrm{dry}}}\left(K_{\mathrm{dry}}, \mu_{\mathrm{dry}}\right) \\
& \quad=\frac{1}{|J(\bar{\phi}, \bar{c})|} f_{\phi, c}(\bar{\phi}, \bar{c}) \\
& \quad=\frac{\phi_{0}}{\left|\mu_{\mathrm{dry}}\left(K_{c}-K_{q}\right)-K_{\mathrm{dry}}\left(\mu_{c}-\mu_{q}\right)\right|} f_{\phi, c}(\bar{\phi}, \bar{c}),
\end{aligned}
$$

where $f_{\phi, c}(\bar{\phi}, \bar{c})$ is the joint distribution of porosity and clay content evaluated in the real roots of the model.

\section{EXAMPLES}

We show here several examples using the equations derived in the section "Methodology." The method can be applied to any equation as long as the rock physics model is differentiable and the PDF of the input random variable is parametric; i.e., it has an analytical closed form. The last two examples show the application of the method to models in a multivariate domain and a real case study with well logs.

\section{Gassmann's equation with uncertain porosity}

By following the same order of the models presented in the section "Methodology," the first example is the estimation of the distribution of the saturated-rock bulk modulus using Gassmann's equation with uncertain porosity (Figure 3 ).

We assume that porosity is distributed according to a Gaussian distribution $\phi \sim N\left(\mu_{\phi}, \sigma_{\phi}^{2}\right)$ with mean $\mu_{\phi}=0.20$ and standard deviation $\sigma_{\phi}=0.05$. The input PDF is shown in Figure 3a. We also assume that $K_{\text {mat }}, K_{\mathrm{fl}}$, and $K_{\text {dry }}$ are constant: $K_{\text {mat }}=30 \mathrm{GPa}$, $K_{\mathrm{fl}}=2.5 \mathrm{GPa}$, and $K_{\mathrm{dry}}=15 \mathrm{GPa}$. This could be the case when we have some laboratory measurements in which we can measure with good precision the elastic moduli but the porosity measurement is uncertain due to the unknown percentage of nonconnected porosity. If these parameters are constant, the behavior of Gassmann's equation (equation 13) is shown in Figure 3b. By applying
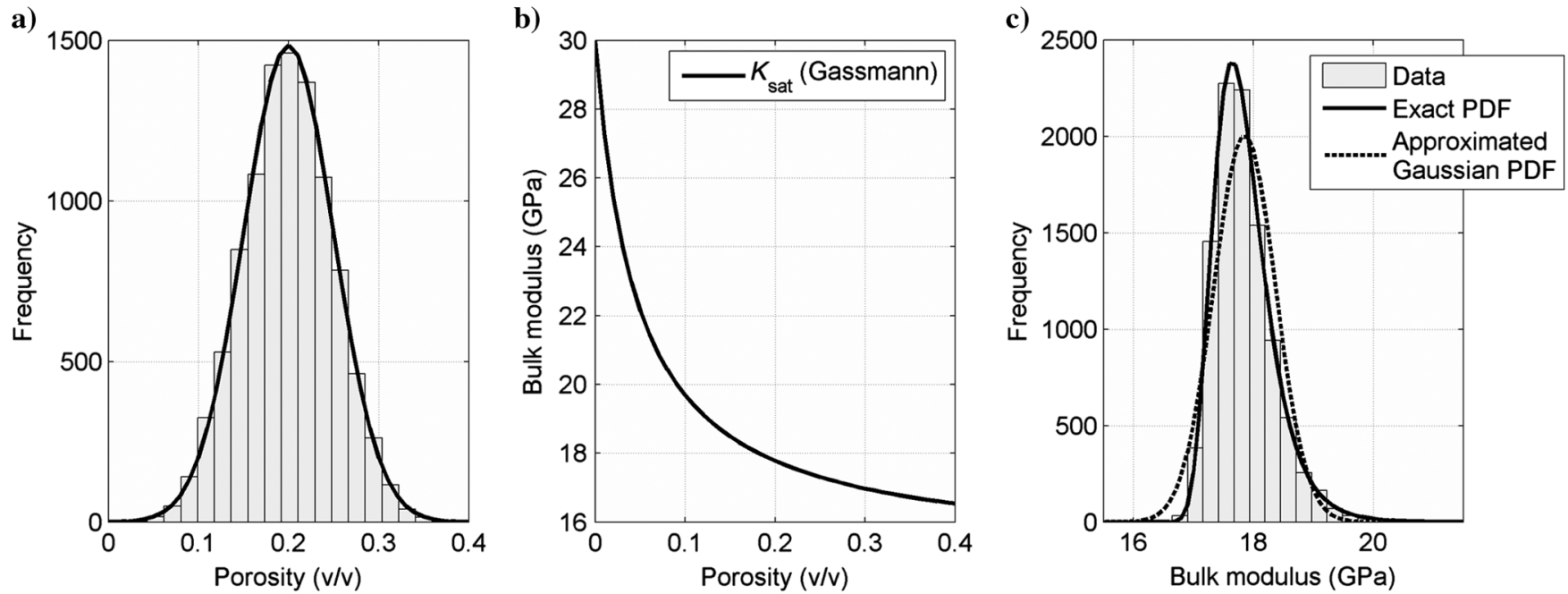

Figure 3. Estimation of the PDF of the saturated-rock bulk modulus using Gassmann's equation with uncertain porosity: (a) input Gaussian distribution of porosity, (b) Gassmann's equation for saturated-rock bulk modulus as a function of porosity, (c) predicted saturated-rock bulk modulus distribution (solid line) and Gaussian approximation (dashed line). Distributions are compared to histograms of 10,000 samples generated through Monte Carlo simulations and are normalized by the bin size for comparison. 
equations 15 and 17, we can compute the exact analytical PDF of the saturated-rock bulk modulus, shown in Figure 3c.

Because Gassmann's equation is not linear, we observe that the so-obtained PDF is not Gaussian, but it is skewed. We then compare the PDF with the histogram of 10,000 samples obtained by Monte Carlo simulations: First we generate 10,000 samples of porosity from the input distribution, then we apply Gassmann's equation, and finally we plot the histogram of the samples of saturated-rock bulk modulus and impose the exact PDF in equation 17. We point out that in this example and in all the following examples, the PDFs are normalized by the bin size to make them comparable to the histograms; in other words, the shape of the PDF is preserved but the integral of the curve is not 1 , but it is equal to the area of the histogram.

In Figure 3, we can appreciate that the output PDF exactly matches the histogram. We can compare the exact PDF with the approximated distribution that we can obtain from a Monte Carlo simulation approach (Figure 3c). For this comparison, we reestimate the output PDF from the 10,000 Monte Carlo samples: In particular, we assume that the output distribution is Gaussian and estimate its parameters from the Monte Carlo samples. Due to the skewness of the output distribution, the Gaussian approximation does not match the exact PDF. The mode is shifted to a value (i.e., the empirical mean) higher than the actual mode of the histogram, and the Gaussian PDF also shows nonzero likelihood values for bulk moduli less than $17 \mathrm{GPa}$, even though the Monte Carlo histogram does not contain these values. These problems are not present in the exact PDF.

In the second example (Figure 4), we still assume the same porosity distribution as in the previous example (Figure 4a), and we assume that $K_{\text {mat }}$ and $K_{\mathrm{fl}}$ are constant, but we now use a rock physics model (Nur's model) for $K_{\text {dry }}$ (equation 18 ) with critical porosity $\phi_{0}=0.42$. By applying equations 19 and 20, we estimate the exact PDF of the saturated-rock bulk modulus. The results are shown in Figure 4: In this case, Gassmann's equation has a more linear behavior (due to the linearization of the dry-bulk modulus with respect to porosity) and the output PDF as a consequence shows a
Gaussian behavior. The approximated Gaussian PDF obtained from the Monte Carlo simulation is in this case a good approximation.

The third example shows the estimated PDF of the dry-rock bulk modulus with uncertain porosity when the bulk moduli of matrix, fluid, and saturated rock are known. This could be the case when we can obtain reliable values of $\mathrm{P}$ - and S-wave velocities and of density from well logs, and we can compute the saturated-rock bulk modulus from them, but the porosity measurements are uncertain, and we want to account for this uncertainty in the estimation of the dry-rock bulk modulus. The dry-bulk modulus can be computed using the inverse Gassmann's equation (equation 14). We assume that porosity is distributed according to a Gaussian distribution $\phi \sim N\left(\mu_{\phi}, \sigma_{\phi}^{2}\right)$ with mean $\mu_{\phi}=0.20$ and standard deviation $\sigma_{\phi}=0.04$. The input PDF is shown in Figure 5a. We also assume that $K_{\text {mat }}, K_{\mathrm{fl}}$, and $K_{\text {sat }}$ are constant: $K_{\text {mat }}=30 \mathrm{GPa}, K_{\mathrm{fl}}=2.5 \mathrm{GPa}$, and $K_{\text {sat }}=18 \mathrm{GPa}$. By applying equations 21 and 23, we can compute the exact PDF of $K_{\text {dry }}$, which is shown in Figure 5c. Once again, we compare the soobtained PDF with the Gaussian approximation, which does not match the exact PDF. The Gaussian approximation is not correct in this case because the mode is lower by almost $1 \mathrm{GPa}$ than the histogram mode. The distribution shows nonzero likelihood values for bulk moduli greater than $17 \mathrm{GPa}$, whereas the exact PDF correctly assigns a likelihood close to zero.

\section{Gassmann's equation with uncertain saturation}

We then perform a similar analysis on Gassmann's equation assuming uncertain water saturation. We assume for simplicity that the fluid consists of two phases: water and gas, but the method could be applied to different saturation scenarios (oil-water or gas-oil-water). In the next examples, we assume that $K_{\text {mat }}, K_{\mathrm{dry}}$, and $\phi$ are known constant values: $K_{\text {mat }}=30 \mathrm{GPa}, K_{\text {dry }}=$ $15 \mathrm{GPa}$, and $\phi=0.3$. We also assume that the bulk moduli of the fluid components are constant: $K_{w}=2.5 \mathrm{GPa}$ and $K_{g}=0.2 \mathrm{GPa}$. We show here four examples: patchy saturation where water saturation is distributed according to a beta distribution, homogeneous saturation where water saturation is distributed according to a beta distribution, patchy saturation where water
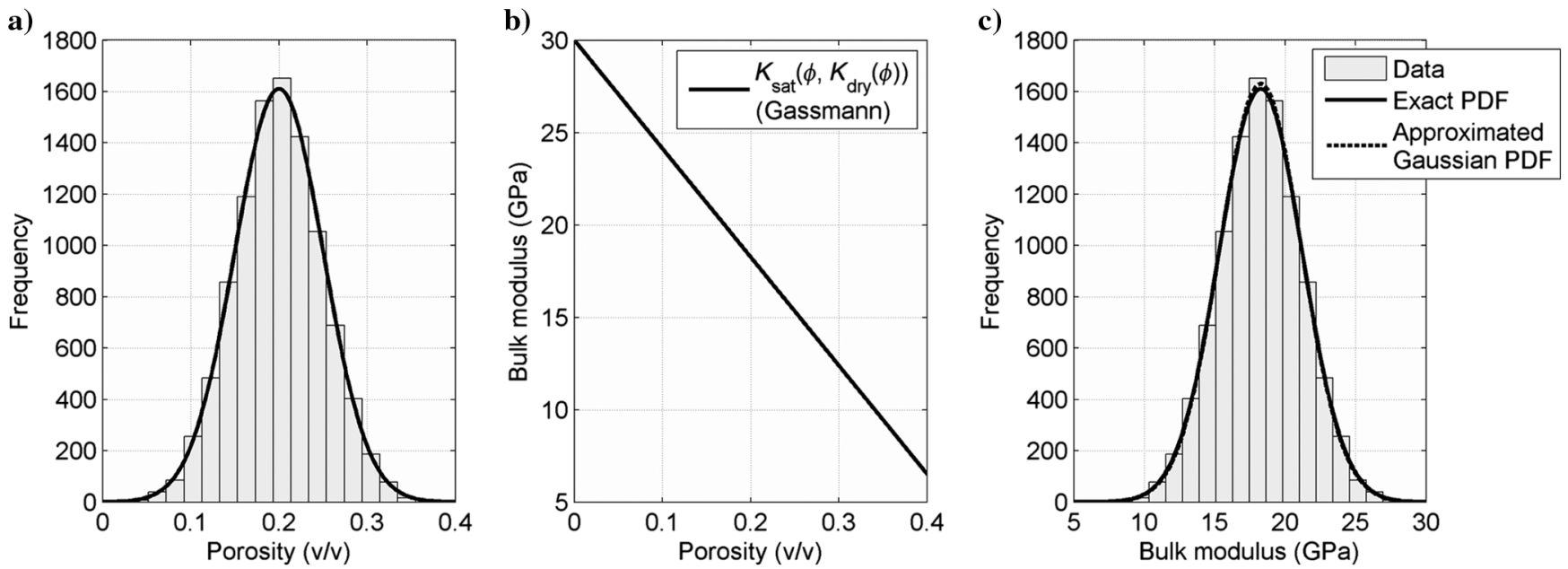

Figure 4. Estimation of the PDF of the saturated-rock bulk modulus using Nur's model and Gassmann's equation with uncertain porosity: (a) input Gaussian distribution of porosity, (b) Nur's model and Gassmann's equation for saturated-rock bulk modulus as a function of porosity, and (c) predicted saturated-rock bulk modulus distribution (solid line) and Gaussian approximation (dashed line). Distributions are compared to histograms of 10,000 samples generated through Monte Carlo simulations and are normalized by the bin size for comparison. 
saturation is distributed according to a uniform distribution, and homogeneous saturation where water saturation is distributed according to a uniform distribution.

We first assume that the fluid distribution is patchy (equation 24), and we assume a beta distribution for water saturation $s_{w} \sim$ $B(0.1,0.1)$. The input distribution of $s_{w}$ is shown in Figure 6a where it is normalized and compared to a histogram of 10,000 samples: The PDF shows as expected two peaks in 0 and 1 . We then apply Gassmann's equation (Figure 6b) and compute the exact PDF of the saturated-rock bulk modulus using equations 25 and 27 . The PDF of $K_{\text {sat }}$ is shown in Figure $6 \mathrm{c}$. The model is almost linear for the patchy saturation assumption (linear average in equation 24), and the output distribution preserves the bimodal feature at the extremes of the interval range. In this case, we do not compare the output PDF with the Gaussian approximation because the behavior of the saturated-rock bulk modulus cannot be described by a Gaussian distribution.

Under the same assumption for the probability distribution of water saturation, we show the same example but with a homogeneous fluid distribution (equation 28). The output distribution is obtained by applying equations 29 and 30 (the explicit equation for a beta distribution is written in equation 31). The results are shown in Figure 7. In Figure 7c, we observe that the output distribution is still similar to a beta, but it is not symmetric even though a)

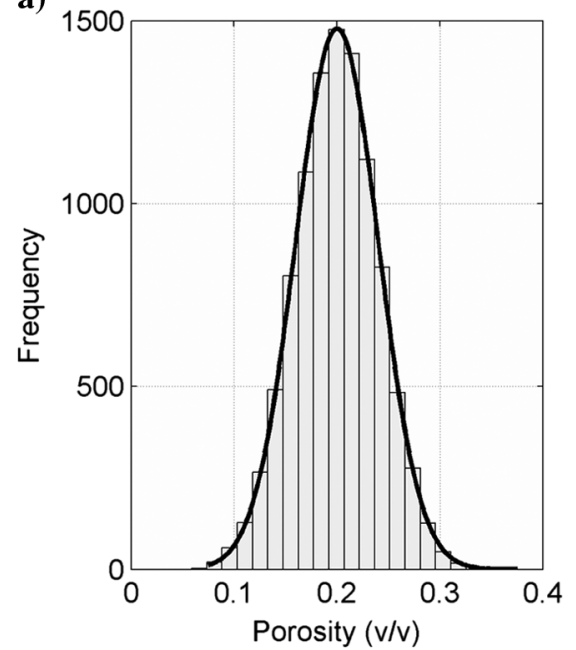

b)

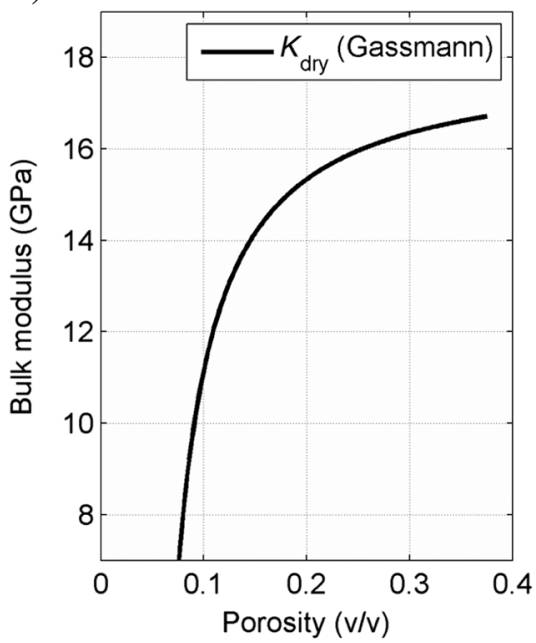

c)

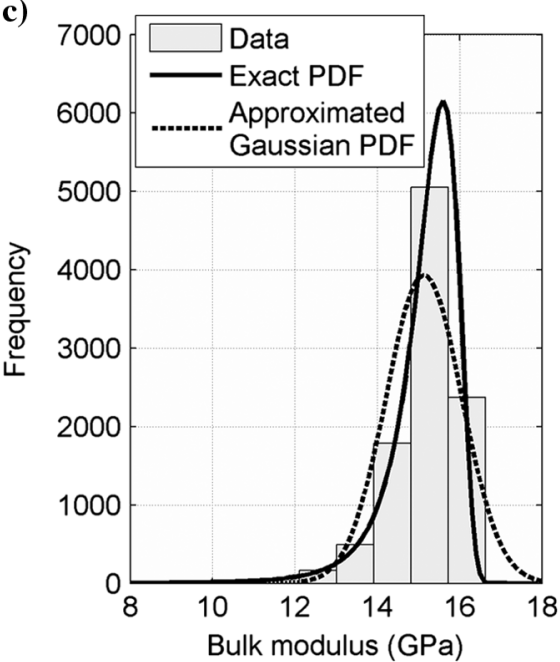

Figure 5. Estimation of the PDF of the dry-rock bulk modulus using Gassmann's equation with uncertain porosity: (a) input Gaussian distribution of porosity, (b) Gassmann's equation for dry-rock bulk modulus as a function of porosity, and (c) predicted dry-rock bulk modulus distribution (solid line) and Gaussian approximation (dashed line). Distributions are compared to histograms of 10,000 samples generated through Monte Carlo simulations and are normalized by the bin size for comparison.

a)

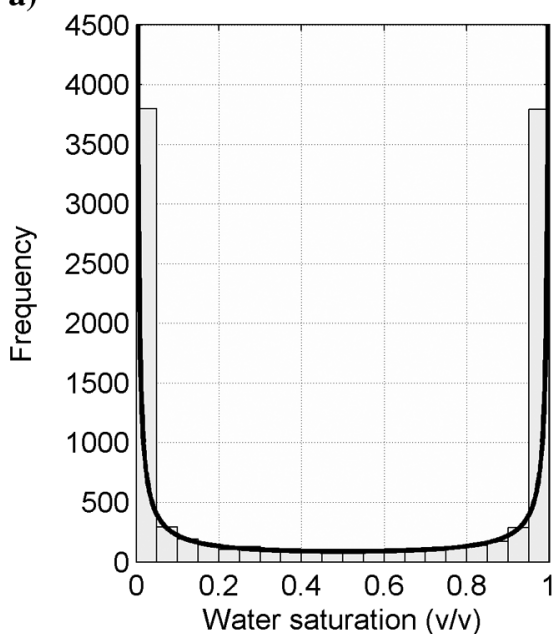

b)

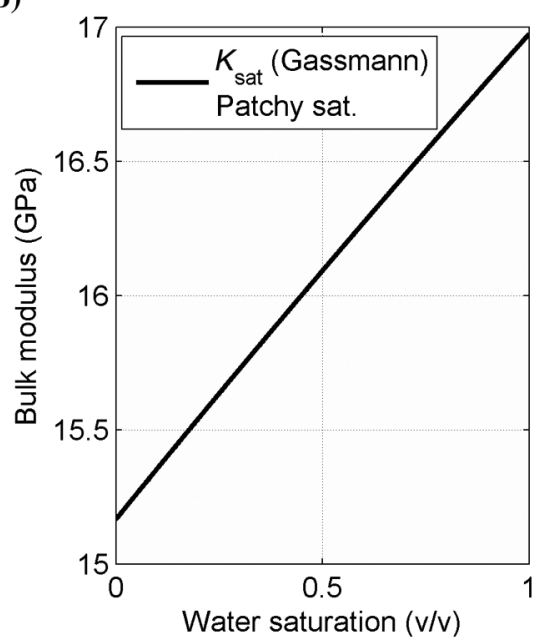

c)

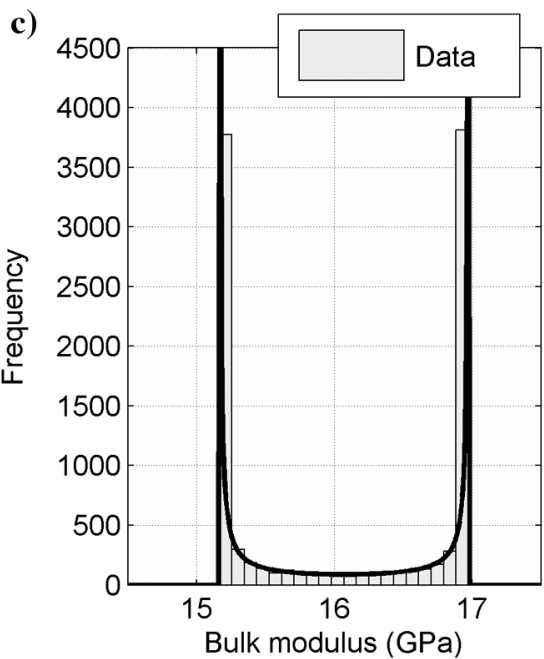

Figure 6. Estimation of the PDF of the saturated-rock bulk modulus using Gassmann's equation with uncertain saturation (patchy saturation): (a) input beta distribution of water saturation, (b) Gassmann's equation for saturated-rock bulk modulus as a function of water saturation, and (c) predicted saturated-rock bulk modulus distribution. Distributions are compared to histograms of 10,000 samples generated through Monte Carlo simulations and are normalized by the bin size for comparison. 
the initial distribution is symmetric. In the output, lower values of the bulk modulus are more likely than higher values due to the nonlinear behavior of Gassmann's equation for homogeneous fluid distribution (Figure $7 b$ ).

We now repeat the same exercise but assume a uniform distribution for water saturation between 0 and 1 . In Figure 8, we show the results for patchy saturation, whereas in Figure 9, we show the results for homogeneous saturation. In both cases, the output PDF exactly matches the corresponding histogram (Figures $8 \mathrm{c}$ and 9c).

\section{Raymer's model with uncertain porosity}

As described in the section "Methodology," the proposed method is not limited to fluid substitution problems. It can be applied to any rock physics model. In the next two examples, we apply the proposed method to an empirical model (Raymer's equation) and to a theoretical model (soft-sand model).

Raymer's model (equation 33) is an empirical equation quadratic in porosity, even though the behavior is almost linear in the usual porosity range ([0, 0.37]). We apply the proposed method to compute the PDF of P-wave velocity with uncertain porosity. We assume that the P-wave velocity of the matrix and the P-wave velocity of the fluid are constant, in our example, $V_{\mathrm{P}}^{\text {mat }}=$ $5000 \mathrm{~m} / \mathrm{s}$ and $V_{\mathrm{P}}^{\mathrm{fl}}=1500 \mathrm{~m} / \mathrm{s}$. We assume that porosity $\phi$ is distributed according to a Gaussian distribution $\phi \sim N\left(\mu_{\phi}, \sigma_{\phi}^{2}\right)$ with mean $\mu_{\phi}=0.20$ and standard deviation $\sigma_{\phi}=0.05$. By applying equations 34 and 36, we estimate the exact PDF of $V_{\mathrm{P}}$. The initial distribution of porosity $\phi$ is shown in Figure 10a, the rock physics
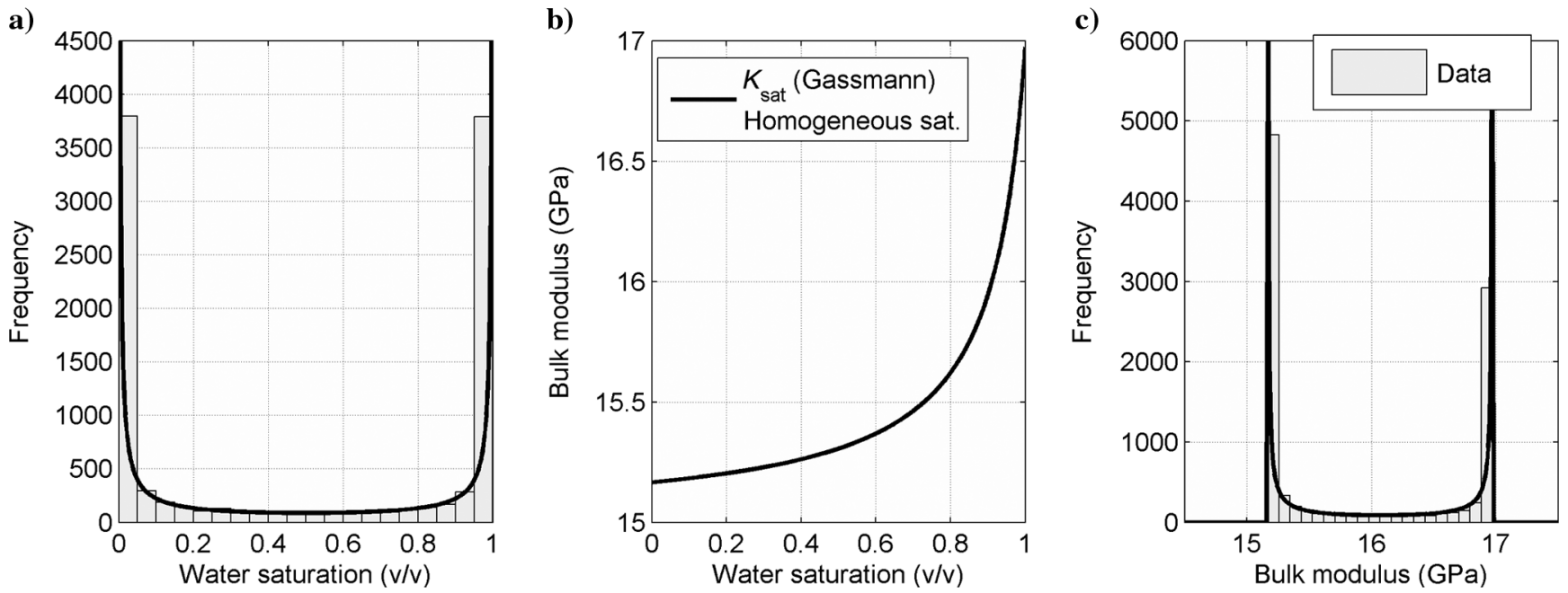

Figure 7. Estimation of the PDF of the saturated-rock bulk modulus using Gassmann's equation with uncertain saturation (homogeneous saturation): (a) input beta distribution of water saturation, (b) Gassmann's equation for saturated-rock bulk modulus as a function of water saturation, and (c) predicted saturated-rock bulk modulus distribution. Distributions are compared to histograms of 10,000 samples generated through Monte Carlo simulations and are normalized by the bin size for comparison.
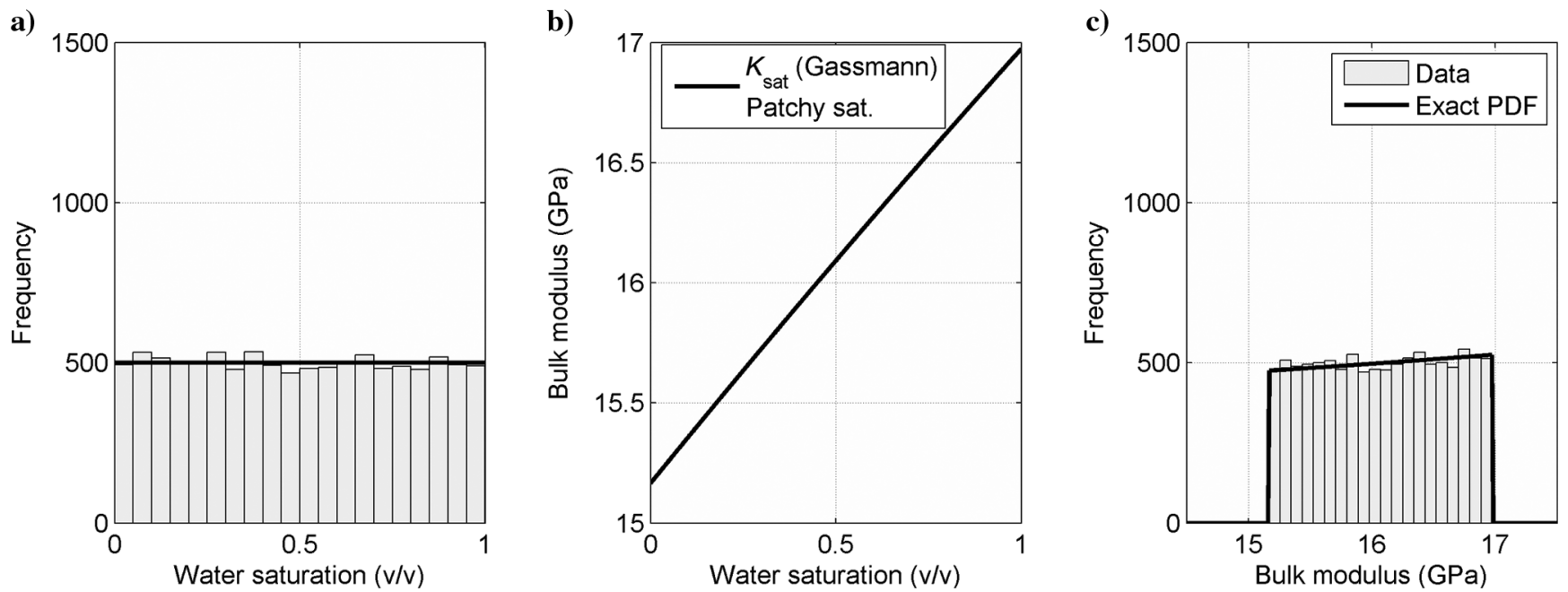

Figure 8. Estimation of the PDF of the saturated-rock bulk modulus using Gassmann's equation with uncertain saturation (patchy saturation): (a) input uniform distribution of water saturation, (b) Gassmann's equation for saturated-rock bulk modulus as a function of water saturation, and (c) predicted saturated-rock bulk modulus distribution. Distributions are compared to histograms of 10,000 samples generated through Monte Carlo simulations and are normalized by the bin size for comparison. 
model in Figure 10b, and the output distribution of $V_{\mathrm{P}}$ in Figure 10c. Because the initial distribution is Gaussian and the model is almost linear, the output PDF is approximately Gaussian.

Similarly, we apply the same approach to Raymer-Dvorkin's $V_{\mathrm{S}}$ prediction model (equation 37 ) under the same porosity assumption $\phi \sim N\left(0.2,0.05^{2}\right)$ and assume a constant S-wave velocity of the matrix $V_{\mathrm{S}}^{\mathrm{mat}}=2500 \mathrm{~m} / \mathrm{s}$. The results are shown in Figure 11 and show a Gaussian linear behavior similar to what we observed for $V_{\mathrm{P}}$.

\section{Soft-sand model with uncertain porosity}

This example is related to the soft-sand model in which we aim to estimate the PDF of the dry-rock elastic moduli with uncertain porosity using Hertz-Mindlin contact theory and Hashin-Shtrikman modified bounds (equations 38 and 39, respectively, for bulk and shear moduli). We assume that all the parameters in equation 40 are constant: $K_{\text {mat }}=30 \mathrm{GPa}, \mu_{\text {mat }}=12 \mathrm{GPa}, K_{\mathrm{HM}}=3 \mathrm{GPa}, \mu_{\mathrm{HM}}=$ $1.5 \mathrm{GPa}$, and $\phi_{0}=0.42$. As in the previous example, porosity is distributed according to a Gaussian distribution $\phi \sim N\left(\mu_{\phi}, \sigma_{\phi}^{2}\right)$ with mean $\mu_{\phi}=0.20$ and standard deviation $\sigma_{\phi}=0.05$.

The output PDFs of the dry-rock elastic moduli are computed by applying equations 41 and 43. Differently from the previous example, the rock physics model (equations 38 and 39) in this case is nonlinear (Figures 12b and 13b); therefore, we expect a nonGaussian behavior in the output PDFs of $K_{\mathrm{dry}}$ and $\mu_{\mathrm{dry}}$. The exact PDF of $K_{\text {dry }}$ is shown in Figure 12c, and the exact PDF of $\mu_{\text {dry }}$ is shown in Figure 13c. Both PDFs show log-normal behavior, and
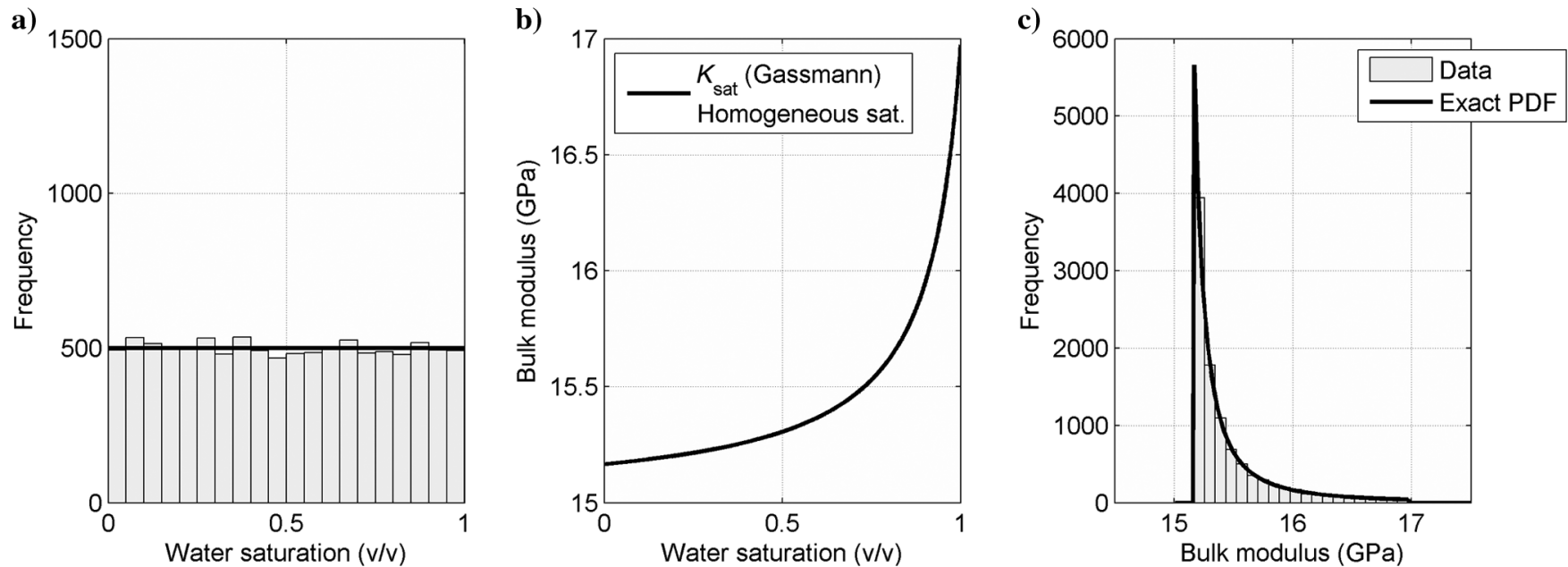

Figure 9. Estimation of the PDF of the saturated-rock bulk modulus using Gassmann's equation with uncertain saturation (homogeneous saturation): (a) input uniform distribution of water saturation, (b) Gassmann's equation for saturated-rock bulk modulus as a function of water saturation, and (c) predicted saturated-rock bulk modulus distribution. Distributions are compared to histograms of 10,000 samples generated through Monte Carlo simulations and are normalized by the bin size for comparison.
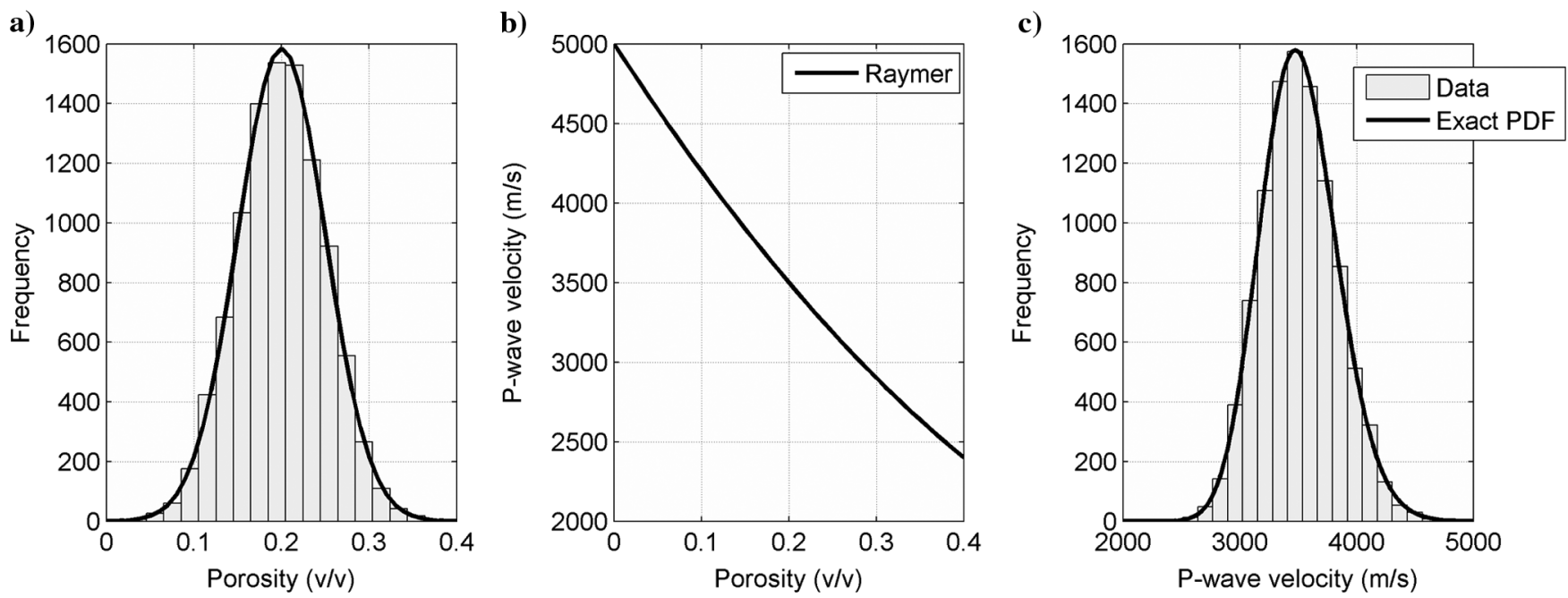

Figure 10. Estimation of the PDF of P-wave velocity using Raymer's equation with uncertain porosity: (a) input Gaussian distribution of porosity, (b) Raymer's equation for P-wave velocity as a function of porosity, and (c) predicted P-wave velocity distribution. Distributions are compared to histograms of 10,000 samples generated through Monte Carlo simulations and are normalized by the bin size for comparison. 
they perfectly match the histograms 10,000 samples generated through Monte Carlo simulations.

\section{Inclusion model with uncertain porosity}

The last example for the univariate case focuses on the estimation of the PDF of the dry-rock elastic moduli with uncertain porosity using the inclusion model proposed by Kuster and Toksöz (1974) shown in equations 44 (implicit formulation) and 45 (explicit formulation). We assume a rock matrix with the following properties: $K_{\text {mat }}=30 \mathrm{GPa}, \mu_{\text {mat }}=12 \mathrm{GPa}$, and one inclusion represented by pore space with spherical pores filled by water. Therefore, in equation $45, N=1$ and $f_{1}=\phi$ and because the fluid within the inclusion is water, the bulk modulus of the inclusion is $K_{1}=2.25 \mathrm{GPa}$, and the shear modulus is $\mu_{1}=0 \mathrm{GPa}$. Porosity is distributed according to a Gaussian distribution $\phi \sim N\left(\mu_{\phi}, \sigma_{\phi}^{2}\right)$ with mean $\mu_{\phi}=0.20$ and standard deviation $\sigma_{\phi}=0.05$.

The output PDFs of the dry-rock elastic moduli are computed by applying equations 47 and 48, and the results are shown in Figures 14 and 15. The rock physics model (equation 45) is almost linear (Figures $14 \mathrm{~b}$ and $15 \mathrm{~b}$ ), and the output PDFs of $K_{\mathrm{KT}}^{*}$ and $\mu_{\mathrm{KT}}^{*}$ approximately preserve the same distribution shape of the porosity distribution. The exact PDF of $K_{\mathrm{KT}}^{*}$ is shown in Figure $14 \mathrm{c}$, and the exact PDF of $K_{\mathrm{KT}}^{*}$ is shown in Figure $15 \mathrm{c}$ and are compared to the Monte Carlo histogram.

\section{Application in multivariate domains}

This example shows the application of the method in a multivariate case. In most of the cases in fact, it is not realistic to assume that
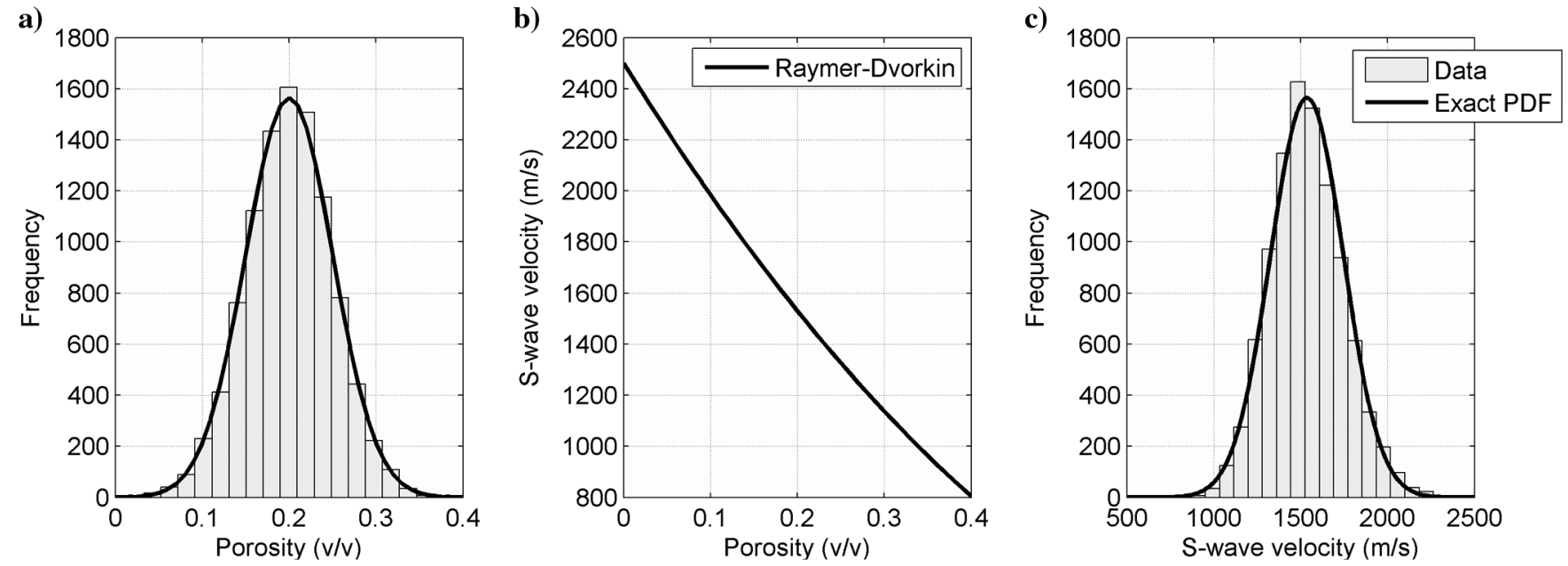

Figure 11. Estimation of the PDF of S-wave velocity using Raymer-Dvorkin's equation with uncertain porosity: (a) input Gaussian distribution of porosity, (b) Raymer-Dvorkin's equation for S-wave velocity as a function of porosity, and (c) predicted S-wave velocity distribution. Distributions are compared to histograms of 10,000 samples generated through Monte Carlo simulations and are normalized by the bin size for comparison.
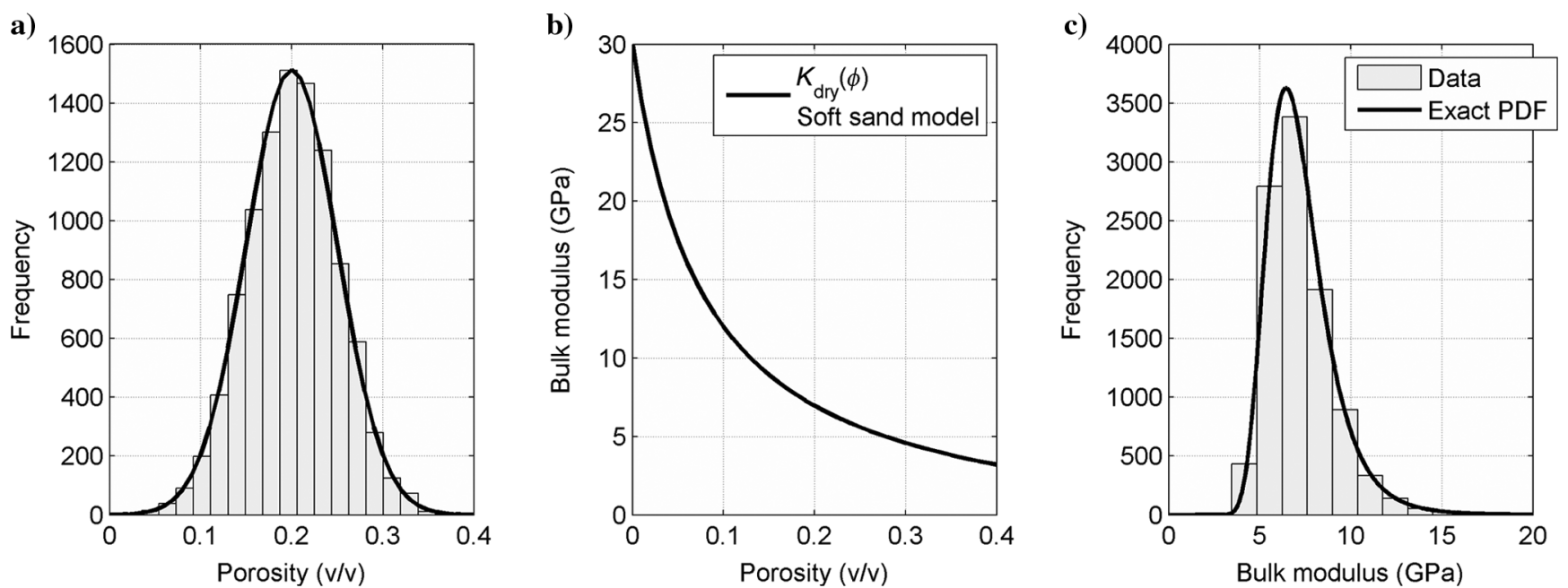

Figure 12. Estimation of the PDF of dry-rock bulk modulus using soft-sand model with uncertain porosity: (a) input Gaussian distribution of porosity, (b) modified Hashin-Shtrikman lower bound for dry-rock bulk modulus as a function of porosity, and (c) predicted dry-rock bulk modulus distribution. Distributions are compared to histograms of 10,000 samples generated through Monte Carlo simulations and are normalized by the bin size for comparison. 
only one variable is uncertain. The mathematical formulation in multivariate domains is more complicated; however, for purposes of illustration, we show the application of the method with Nur's model, which is a model of two functions. In our example, we assume two random variables, porosity and clay volume, jointly distributed according to a bivariate Gaussian distribution with mean $(0.20,0.40)$, standard deviation $(0.03,0.05)$, and correlation -0.8 . When the distribution is skewed, lognormal or closed-skew Gaussian distributions (Karimi et al., 2010) can be used to describe the nonsymmetric behavior. When the distribution is not unimodal, Gaussian mixture models (i.e., linear combination of Gaussian distributions; see Grana and Della Rossa, 2010) can be used to describe the multimodal behavior.
We then apply Nur's model to compute the posterior distribution of two variables: the bulk and shear moduli of the dry rock. We assume that the rock is made by two mineralogical components: quartz and clay with the following properties: $K_{q}=36 \mathrm{GPa}$, $\mu_{q}=45 \mathrm{GPa}, K_{c}=21 \mathrm{GPa}, \mu_{c}=7 \mathrm{GPa}$. The mixture of the mineralogical components is described using the Voigt average.

In Figure 16, we show the joint distribution of porosity and clay content with the corresponding marginal distributions, the analytical distribution of bulk and shear moduli of the dry rock with the corresponding marginal distributions, and the comparison with the simulated Monte Carlo samples. The comparison with the approximated Gaussian PDF extracted from the Monte Carlo simulation is shown in Figure 17. If we assume a bivariate Gaussian PDF to a)

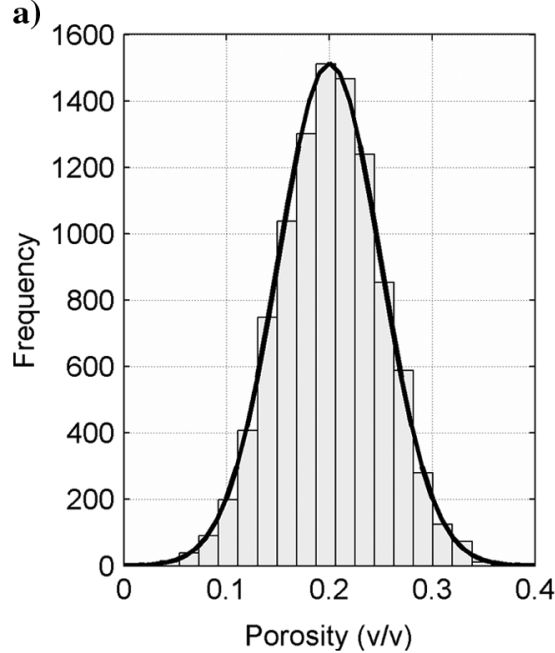

b)

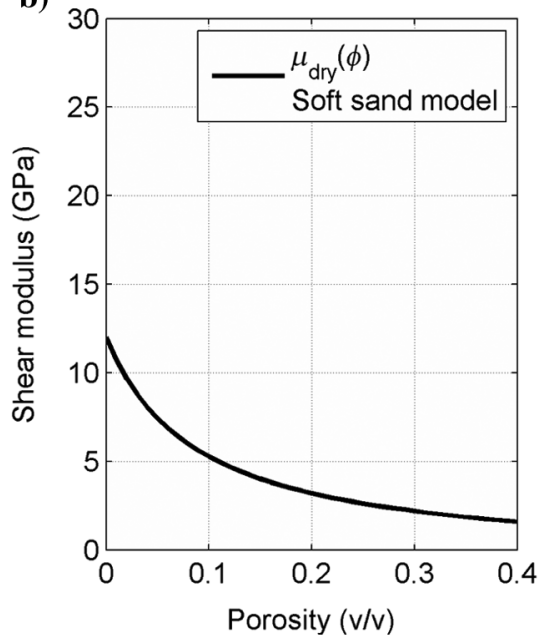

c)

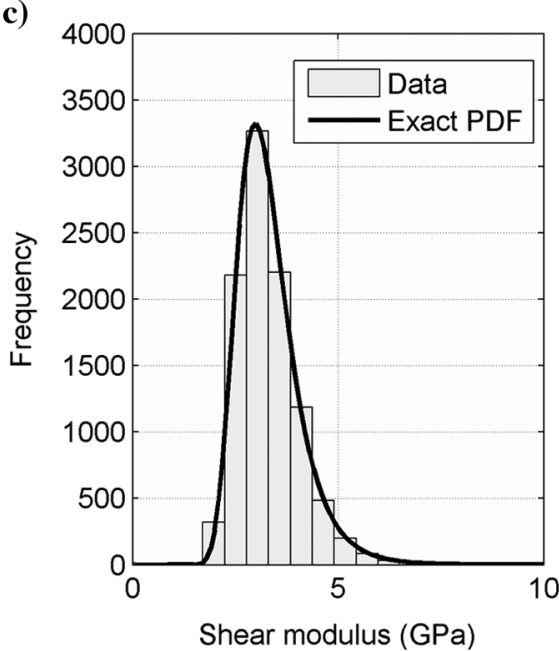

Figure 13. Estimation of the PDF of dry-rock shear modulus using soft-sand model with uncertain porosity: (a) input Gaussian distribution of porosity, (b) modified Hashin-Shtrikman lower bound for dry-rock shear modulus as a function of porosity, and (c) predicted dry-rock shear modulus distribution. Distributions are compared to histograms of 10,000 samples generated through Monte Carlo simulations and are normalized by the bin size for comparison.
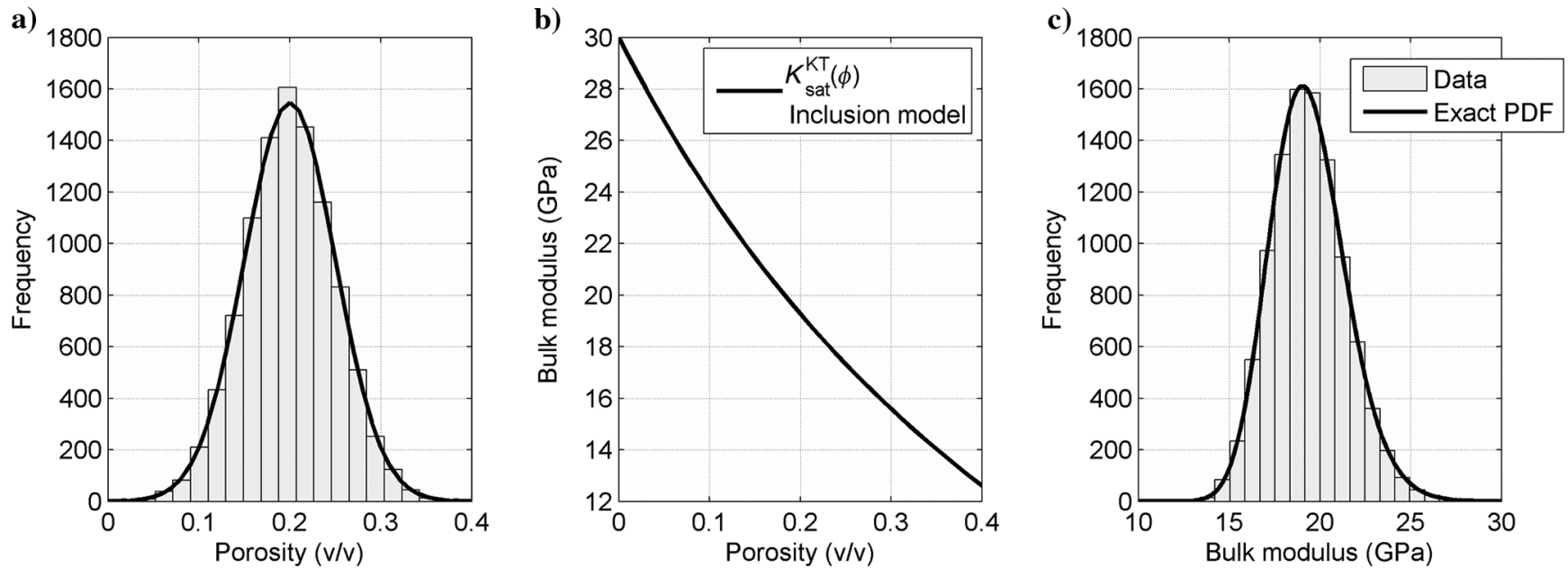

Figure 14. Estimation of the PDF of water saturated-rock bulk modulus using an inclusion model with uncertain porosity: (a) input Gaussian distribution of porosity, (b) Kuster-Toksöz model for the water-saturated-rock bulk modulus as a function of porosity, and (c) predicted water saturated-rock bulk modulus distribution. Distributions are compared to histograms of 10,000 samples generated through Monte Carlo simulations and are normalized by the bin size for comparison. 
approximate the distribution of Monte Carlo samples, we honor the correlation of the exact PDF but not the skewness of the distribution.

\section{Application to well $\operatorname{logs}$}

We finally present the application of the methodology to well-log data analysis. The well logs in this example have been measured in a well of a reservoir with a complex mineralogical composition. The main reservoir is mainly a shaley reservoir with a variable percentage of muscovite and calcite at different levels. The reser- voir fluid phases are water and gas. Well-log data are shown in Figure 18.

In this section, we propose a one-way sensitivity analysis of the uncertainty in the petrophysical curves performed in formation evaluation analysis and the effect on rock physics model predictions. The rock physics model chosen for this study is Raymer's model for P-wave velocity prediction, Raymer-Dvorkin's model for S-wave velocity prediction, and a linear average for density. This model was chosen for the high linear correlation between porosity and elastic properties. In the following paragraphs, we analyze the effect of uncertainty in porosity and saturation.
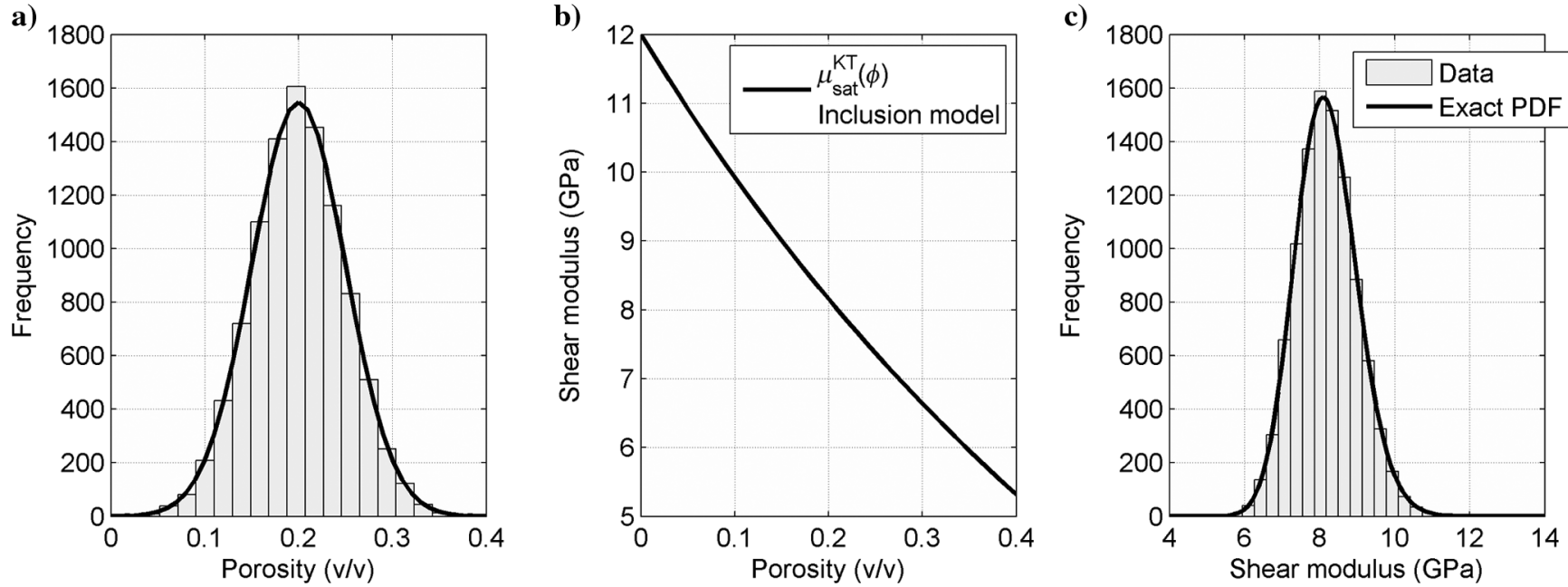

Figure 15. Estimation of the PDF of water saturated-rock shear modulus using an inclusion model with uncertain porosity: (a) input Gaussian distribution of porosity, (b) Kuster-Toksöz model for water saturated-rock shear modulus as a function of porosity, and (c) predicted water saturated-rock shear modulus distribution. Distributions are compared to histograms of 10,000 samples generated through Monte Carlo simulations and are normalized by the bin size for comparison.

a)

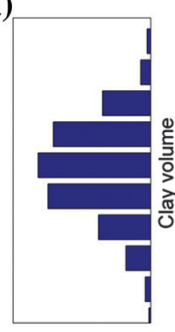

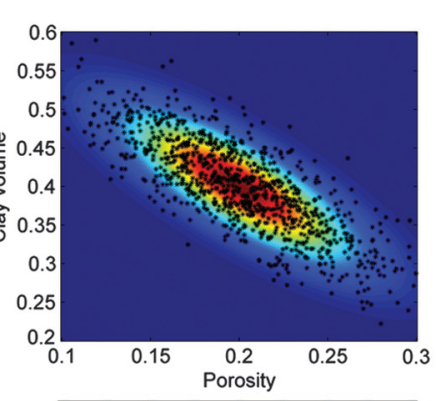

b)

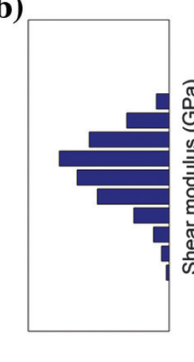

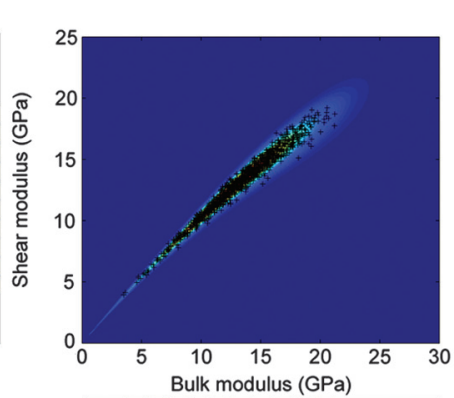

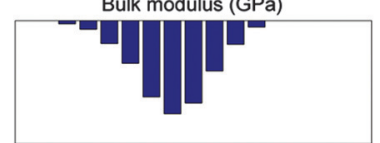

c)
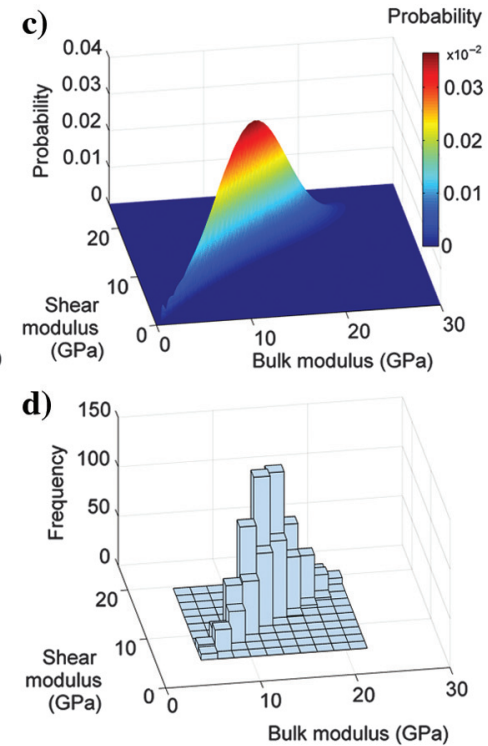

Figure 16. Estimation of the PDF of dry-rock bulk and shear modulus using Nur's critical porosity model with uncertain porosity and clay volume: (a) input bivariate Gaussian distribution of porosity and clay volume with marginal distributions and (b) predicted distribution of dryrock bulk and shear moduli. Black crosses represent 1000 Monte Carlo simulated samples. The comparison between the analytical bivariate distribution and the 2D histogram of Monte Carlo simulated samples in shown in panels (c and d). 
In Figure 19, we show the application for uncertainty propagation in porosity. For each sample of the well log included in the interval corresponding to the main reservoir level, we assumed a Gaussian distribution with mean equal to the actual value of the porosity curve and a constant standard deviation equal to 0.03 . We then applied the presented methodology to the so-computed distributions along the well log: Density is calculated using a linear average with respect to porosity, P-wave velocity is estimated using Raymer's model, and S-wave velocity is computed using Raymer-Dvorkin's model. The local PDFs are shown in Figure 19d-19f.

A similar analysis has been performed for water saturation (Figure 20). In this case, we assumed a uniform distribution with constant support equal to 0.5 , centered around the actual value of the saturation curve and truncated to avoid nonphysical values outside the range $[0,1]$. The same model described above has been applied with the proposed probabilistic approach, and the so-obtained PDFs are shown in Figure 20. For simplicity of illustration, in Figure 20 we only showed the support of the distributions rather than the full PDFs.

\section{DISCUSSION}

The methodology presented in this paper contains the exact probabilistic formulation of the posterior distribution of the variable predicted by a rock physics model in which one of the input variables is uncertain. The probabilistic formulation can be complicated because it requires the solution of a nonlinear equation and the derivative of the rock physics model. However, when we assume that the other properties that appear in the rock physics model are constant, the analytical derivation is straightforward, as shown in several examples in the section "Methodology." When two or more properties are random variables (i.e., uncertain), the input distribution can be described by a joint distribution (as shown in the multivariate example) to describe the distribution of the input variables together with the correlation between these variables. The derivation of the output distribution requires the solution of a system of equations (equation 11) and the computation of partial derivatives (equation 12), which could be complicated, especially for highly nonlinear models. On the other hand, if the joint output distribution can be
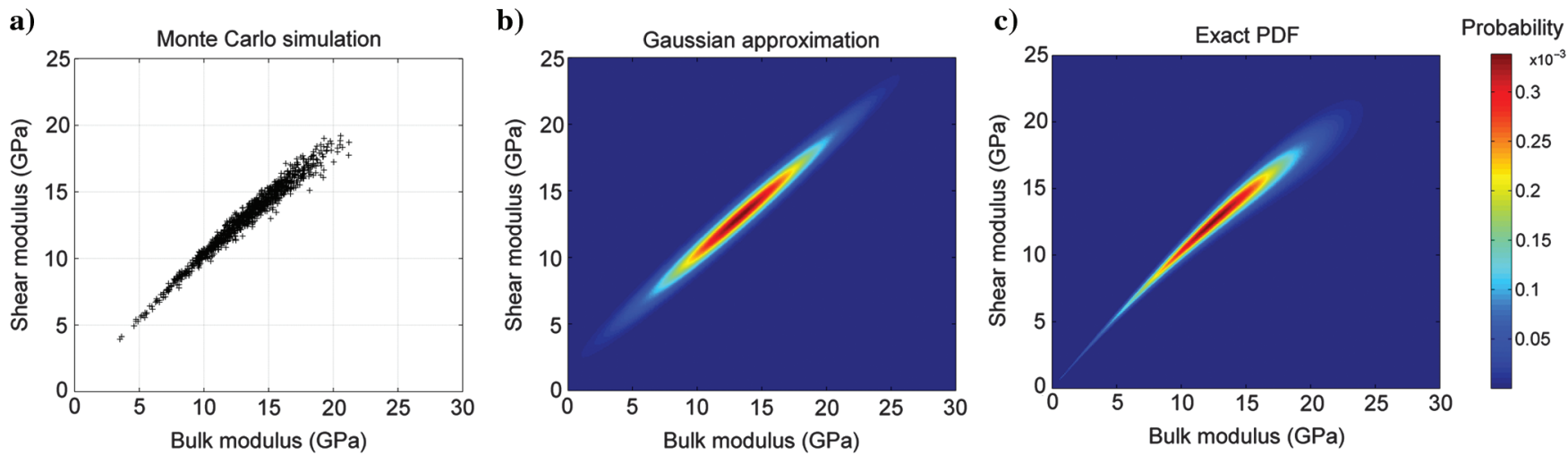

Figure 17. Comparison between exact analytical PDF (c) with Monte Carlo simulation (a) and Gaussian approximated PDF (b).
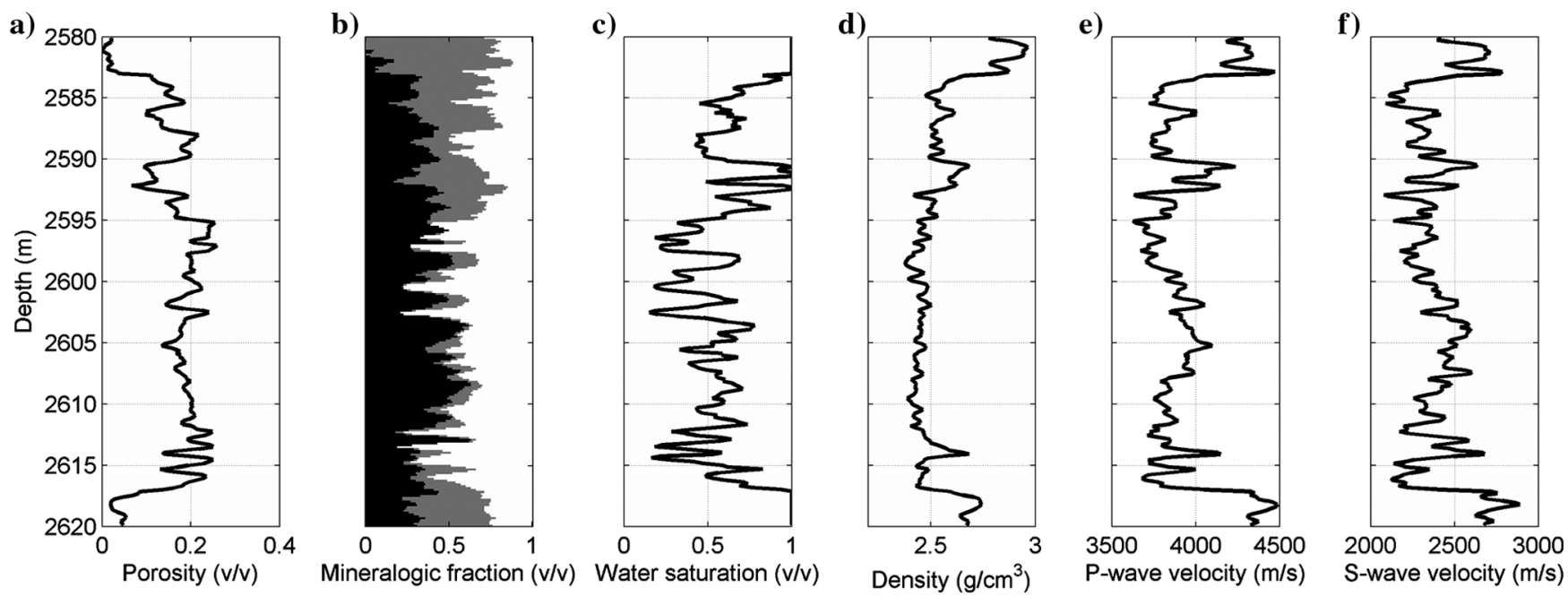

Figure 18. Well-log data: (a) porosity, (b) mineralogical fraction (quartz in black, clay in gray, and silt in white), (c) water saturation, (d) density, (e) P-wave velocity, and (f) S-wave velocity. 
derived, the correlation of rock physics predictions can be estimated without approximations.

The method consists of three steps: the probabilistic description of the input random variable, the computation of the inverse function and of the derivative of the rock physics model, and the calculation of the PDF of the function of the initial random variable.

The PDF of the input random variable can be estimated from actual measurements or data (well logs or laboratory measures) or assumed based on geologic information, nearby fields, or theoretical models. The key point to describe the PDF of the input variable is the assumption related to the shape of the distribution. If we know the most likely value and we have a measure of the uncertainty of this value, the Gaussian distribution can be a good choice. For example, if we know that the porosity of the rock is likely to be 0.2 but this value is uncertain and the range of possible value is between 0.1 and 0.3 , then we can use a Gaussian distribution with mean equal to
0.2 and standard deviation equal 0.03 . However, it is important to point out that the Gaussian distribution is defined and positive for all the real values, which means that the probability of obtaining values less than 0 or greater than 1 is nonzero. If the standard deviation is small enough, these values are negligible, and we can still use the Gaussian PDF. Otherwise, we should truncate the tails of the Gaussian distribution, set the PDF values outside the physical range equal to 0 , and normalize the PDF to make the integral equal to 1. To avoid truncations in the input and output distributions, instead of a Gaussian distribution, we could assume a triangular distribution, which by definition is zero outside of the range. In our example, the triangular distribution would be defined in the interval [0.1, 0.3 ] and symmetric with mode 0.2 .

Some properties, for example, saturations and permeability, cannot be described by a Gaussian distribution. For permeability, it is a common practice to use the logarithm of the permeability rather

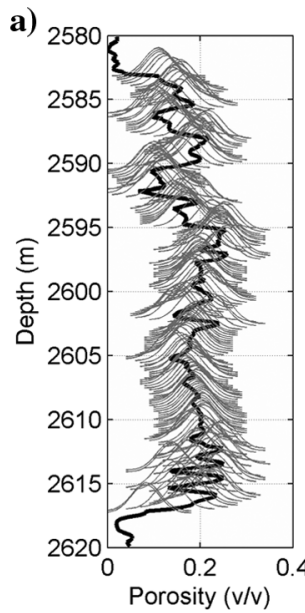

b)

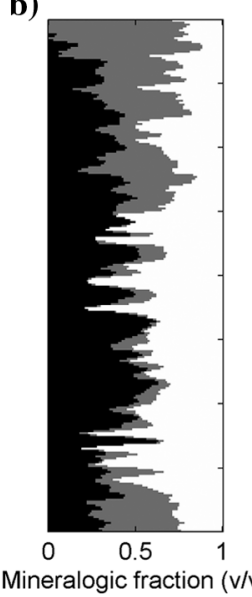

c)

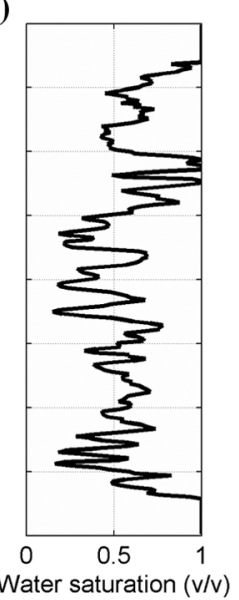

d)

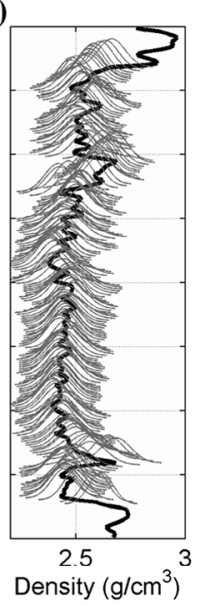

e)

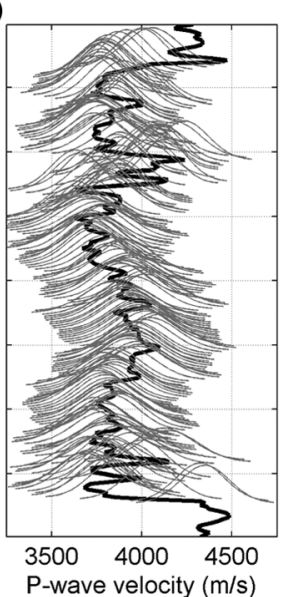

f)

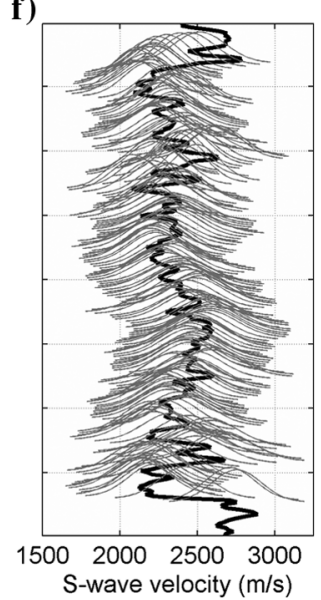

Figure 19. Well-log data application: uncertainty propagation from porosity distributions through Raymer-Dvorkin's model for velocities and linear average for density: (a) porosity (porosity Gaussian distributions are shown in gray in the reservoir zone), (b) mineralogical fraction (quartz in black, clay in gray, and silt in white), (c) water saturation, (d) density, (e) P-wave velocity, and (f) S-wave velocity. The PDFs of the rock physics model predictions are shown in gray.

a)

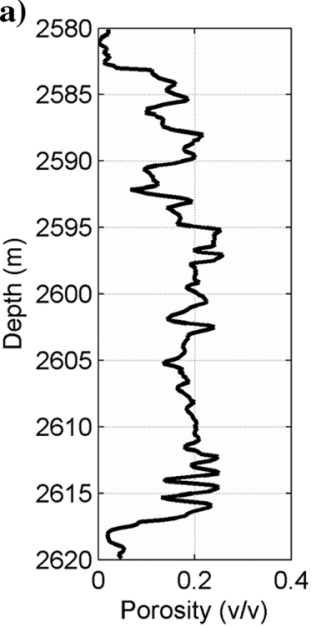

b)

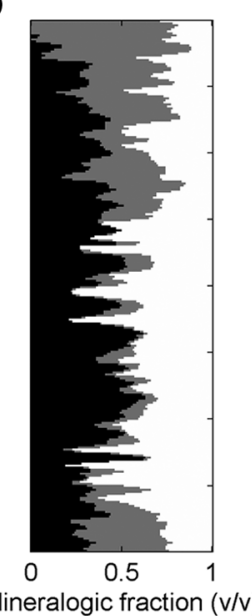

c)

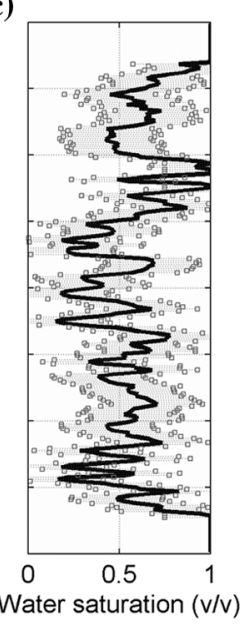

d)

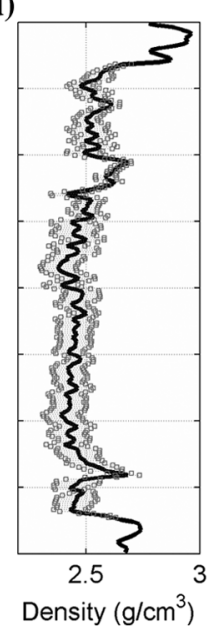

e)

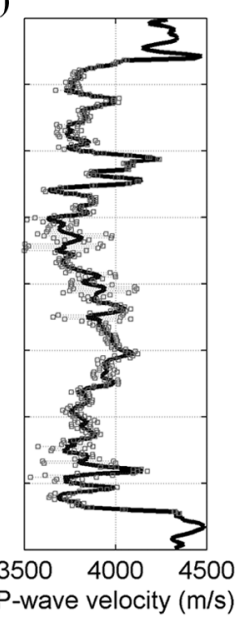

f)

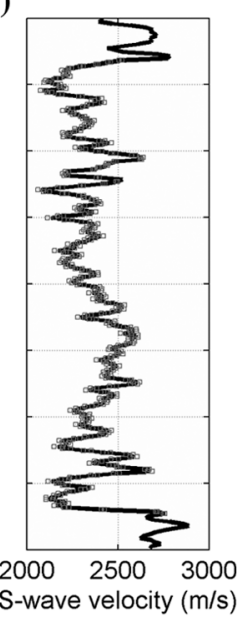

Figure 20. Well-log data application: uncertainty propagation from saturation distributions through Raymer-Dvorkin's model for velocities and linear average for density: (a) porosity, (b) mineralogical fraction (quartz in black, clay in gray, and silt in white), (c) water saturation (saturation uniform distributions are shown in gray in the reservoir zone), (d) density, (e) P-wave velocity, and (f) S-wave velocity. The PDFs of the rock physics model predictions are shown in gray. 
than the permeability. Therefore, we can use a Gaussian distribution for the logarithm of the permeability, which implies that permeability is distributed according to a log-normal distribution. For saturation, the PDF is generally more complicated to describe by parametric distributions for different reasons. First, saturation values are difficult to measure, and the uncertainty is generally high especially for patchy saturation. In this case, a uniform distribution can be the best choice because there are no assumptions on the shape of the distribution and all the values in the saturation range have the same likelihood value. Then, most of the measurements, especially for homogeneous saturation, show that it is unlikely to observe a mixture of $50 \%$ water and $50 \%$ hydrocarbon; therefore, the distribution often shows two peaks close to $0 \%$ and $100 \%$. In other words, the distribution looks like a uniform distribution with two probability peaks at the extremes of the interval. This distribution can be analytically described with a beta distribution, and it can be a good choice for homogeneous fluid distribution scenarios.

The presented method is a valid alternative to Monte Carlo simulations. As a matter of fact, Monte Carlo simulation is a useful tool to generate training data set. In rock physics, this tool is commonly used because the rock physics models do not contain complicated mathematical operations and the application of the forward model is generally straightforward. Therefore, the computational cost of a Monte Carlo simulation is not large. However, to approximate the correct distribution, a large number of samples is still required (for instance, in the comparison in our examples, we used 10,000 samples). Another limitation is that Monte Carlo simulations only provide a training data set. They do not provide an analytical expression for the corresponding PDF. We showed in the first examples that if we want to estimate the posterior distribution from the training data set without knowing the correct shape of the distribution, we then have to make assumptions, for example, the Gaussian distribution of the posterior, and the result might not match the data exactly as the proposed analytical method does. The main advantage of this method is that the expression of the PDF of the predictions is analytical and can be used in probabilistic inversion workflows: Bayesian elastic inversion and Bayesian petrophysical inversion, for example. On the other hand, the method includes several mathematical (analytical) computations to derive the closed form of the posterior. The examples show that if the rock physics model is a function of only one random variable, then the analytical derivation can be achieved in three steps. In one of the examples, the Gassmann's fluid equation with uncertain porosity and uncertain dry-rock bulk modulus, we assumed that two variables where uncertain and we used another rock physics model to describe one of these two variables (dry-rock bulk modulus) as a function of the other one (porosity). This is often the case in rock physics because most of the presented models link rock properties to elastic properties and different equations can be used to express some of the properties involved in the model as a function of the initial rock properties. For example, we expressed the fluid bulk modulus as a function of water saturation. Density could be written in terms of porosity, and the matrix bulk modulus could be described as a function of the clay content or any other mineralogic fraction of the rock.

If we want to study the effect of the uncertainty of two different properties, for example, saturation and porosity, we first should study the effect of the two properties independently and then study the combined effect. A probabilistic formulation for functions of two random variables has been presented in the section "Method- ology" (following the probabilistic formulation in Papoulis, 1984), and it can be easily extended to the multivariate case with more than two variables. However, we want to point out that the analytical expression can become very complicated due to the presence of multiple partial derivatives. Approximations, based on Taylor's expansions, are available as well. This formulation accounts for the correlation between input variables and also the correlation between output predictions. For example, saturation and porosity can be assumed independent, whereas clay content and porosity are generally negatively correlated (the higher the clay content, the lower the porosity) as we can observe in shaley sand dispersed models, for example (see Mavko et al., 2009). This correlation is described by joint distributions as shown in the multivariate example.

If we want to use the statistical rock physics model in inverse problems, such as petrophysical estimation of rock properties, the analytical formulation can provide an advantage. When the analytical formulation is too complicated, Monte Carlo simulations could be used to approximate the output distribution. The tradeoff between the analytical formulation of the exact solution versus numerical approximations of complicated analytical formulations should be established, case by case, depending on the goal of the application, the uncertainty in the input data, and the complexity of the rock physics model.

We finally point out that the methodology has been presented for simplicity of illustration assuming an initial distribution with given mean and variance. However, it can be applied to several distributions with variable means and variances. For example, if we want to apply the method to a well log that is supposed to be uncertain, we can create a set of distributions with locally varying mean (and if necessary, variance) and apply the methodology point by point to obtain a set of distributions that describe the uncertainty of the predictions obtained using a rock physics model from each sample of the initial $\log$.

\section{CONCLUSION}

We presented a new methodology for uncertainty quantification in rock physics model predictions. The method is a probabilistic approach to the forward rock physics model, which provides the exact PDF of the rock physics model predictions given an uncertain input, for example, uncertain porosity in velocity prediction or uncertain saturation in fluid substitution. The method provides a rigorous probabilistic workflow that can be applied to any rock physics model, with any input parametric distribution, and it allows us to overcome the Gaussian assumption generally used in Monte Carlo simulations. The PDF of the rock physics model can then be used in inversion workflows to estimate the rock property distributions and the rock property most likely values, given the elastic property distributions. The methodology has been illustrated with different examples in which we used empirical and theoretical rock physics models and different parametric distributions to describe the rock and fluid property behavior.

\section{ACKNOWLEDGMENTS}

The author acknowledges the School of Energy Resources, the Department of Geology and Geophysics, and the Department of Chemical and Petroleum Engineering of the University of Wyoming for the support. 


\section{APPENDIX A}

\section{MATHEMATICAL DERIVATION}

In this appendix, we show the derivation of equations 16 and 17.

We first derive equation 16 by using the rule of the derivative of the quotient:

$$
\frac{d}{d x} \frac{f(x)}{g(x)}=\frac{f^{\prime}(x) g(x)-f(x) g^{\prime}(x)}{g(x)^{2}} .
$$

By applying this rule to equation 13 , we obtain

$$
\begin{aligned}
& \frac{d}{d \phi} K_{\mathrm{sat}}(\phi) \\
& =\frac{0-\left(1-\frac{K_{\mathrm{dry}}}{K_{\mathrm{mat}}}\right)^{2}\left(\frac{1}{K_{\mathrm{fl}}}-\frac{1}{K_{\mathrm{mat}}}\right)}{\left[\frac{\phi}{K_{\mathrm{fl}}}+\frac{1-\phi}{K_{\mathrm{mat}}}-\frac{K_{\mathrm{dry}}}{K_{\mathrm{mat}}^{2}}\right]} \\
& =-\frac{\left(1-\frac{K_{\mathrm{dry}}}{K_{\mathrm{mat}}}\right)^{2}\left(\frac{1}{K_{\mathrm{fl}}}-\frac{1}{K_{\mathrm{mat}}}\right)}{\left[\phi\left(\frac{1}{K_{\mathrm{fl}}}-\frac{1}{K_{\mathrm{mat}}}\right)+\left(\frac{1-\frac{K_{\mathrm{dry}}}{K_{\mathrm{mat}}}}{K_{\mathrm{mat}}}\right)\right]^{2}} \\
& =-\frac{\left(1-\frac{K_{\mathrm{dry}}}{K_{\mathrm{mat}}}\right)^{2}\left(\frac{K_{\mathrm{mat}}-K_{\mathrm{fl}}}{K_{\mathrm{fl}} K_{\mathrm{mat}}}\right)}{\left[\phi\left(\frac{1}{K_{\mathrm{fl}}}-\frac{1}{K_{\text {mat }}}\right)+\left(\frac{\frac{1-K_{\text {dry }}}{K_{\mathrm{mat}}}}{K_{\text {mat }}}\right)\right]^{2}} \\
& =-\frac{\left(1-\frac{K_{\mathrm{dry}}}{K_{\mathrm{mat}}}\right)^{2}\left(K_{\mathrm{mat}}-K_{\mathrm{fl}}\right)}{K_{\mathrm{fl}} K_{\mathrm{mat}}\left[\phi\left(\frac{1}{K_{\mathrm{fl}}}-\frac{1}{K_{\text {mat }}}\right)+\left(\frac{\frac{1-K_{\mathrm{dry}}}{K_{\mathrm{mat}}}}{K_{\text {mat }}}\right)\right]^{2}} \\
& =-\frac{\left(\frac{K_{\mathrm{mat}}-K_{\mathrm{dry}}}{K_{\mathrm{mat}}}\right)^{2}\left(K_{\mathrm{mat}}-K_{\mathrm{fl}}\right)}{K_{\mathrm{fl}} K_{\mathrm{mat}}\left[\phi\left(\frac{1}{K_{\mathrm{fl}}}-\frac{1}{K_{\mathrm{mat}}}\right)+\left(\frac{\frac{1-K_{\mathrm{dry}}}{K_{\mathrm{mat}}}}{K_{\mathrm{mat}}}\right)\right]^{2}} \\
& =-\frac{\left(K_{\mathrm{mat}}-K_{\mathrm{dry}}\right)^{2}\left(K_{\mathrm{mat}}-K_{\mathrm{fl}}\right)}{K_{\mathrm{fl}} K_{\mathrm{mat}} K_{\mathrm{mat}}^{2}\left[\phi\left(\frac{1}{K_{\mathrm{fl}}}-\frac{1}{K_{\mathrm{mat}}}\right)+\left(\frac{1-\frac{K_{\mathrm{dry}}}{K_{\mathrm{mat}}}}{K_{\mathrm{mat}}}\right)\right]^{2}} \\
& =--\left(K_{\mathrm{mat}}-K_{\mathrm{dry}}\right)^{2}\left(K_{\mathrm{mat}}-K_{\mathrm{fl}}\right) \\
& K_{\mathrm{fl}} K_{\mathrm{mat}} K_{\mathrm{mat}}^{2}\left[\phi\left(\frac{K_{\mathrm{mat}}-K_{\mathrm{fl}}}{K_{\mathrm{mat}}}\right)+\left(\frac{\frac{K_{\mathrm{mat}}-K_{\mathrm{dry}}}{K_{\mathrm{mat}}}}{K_{\mathrm{mat}}}\right)\right]^{2} \\
& =-\frac{\left(K_{\mathrm{mat}}-K_{\mathrm{dry}}\right)^{2}\left(K_{\mathrm{mat}}-K_{\mathrm{fl}}\right)}{K_{\mathrm{fl}} K_{\mathrm{mat}} K_{\mathrm{mat}}^{2}\left[\frac{\phi K_{\mathrm{mat}}\left(K_{\mathrm{mat}}-K_{\mathrm{fl}}\right)+K_{\mathrm{fl}}\left(K_{\mathrm{mat}}-K_{\mathrm{dry}}\right)}{K_{\mathrm{fl}} K_{\mathrm{mat}}^{2}}\right]^{2}} \\
& =-\frac{\left(K_{\mathrm{mat}}-K_{\mathrm{dry}}\right)^{2}\left(K_{\mathrm{mat}}-K_{\mathrm{fl}}\right)}{K_{\mathrm{fl}} K_{\mathrm{mat}} K_{\mathrm{mat}}^{2}\left[\frac{\phi K_{\mathrm{mat}} K_{\mathrm{mat}}-\phi K_{\mathrm{mat}} K_{\mathrm{fl}}+K_{\mathrm{fl}} K_{\mathrm{mat}}-K_{\mathrm{fl}} K_{\mathrm{dry}}}{K_{\mathrm{fl}} K_{\mathrm{mat}}^{2}}\right]^{2}} \\
& =-\frac{K_{\mathrm{fl}}^{2} K_{\mathrm{mat}}^{2} K_{\mathrm{mat}}^{2}\left(K_{\mathrm{mat}}-K_{\mathrm{dry}}\right)^{2}\left(K_{\mathrm{mat}}-K_{\mathrm{fl}}\right)}{K_{\mathrm{fl}} K_{\mathrm{mat}} K_{\mathrm{mat}}^{2}\left[\phi K_{\mathrm{mat}} K_{\mathrm{mat}}-\phi K_{\mathrm{mat}} K_{\mathrm{fl}}+K_{\mathrm{fl}} K_{\mathrm{mat}}-K_{\mathrm{fl}} K_{\mathrm{dry}}\right]^{2}} \\
& =-\frac{K_{\mathrm{mat}} K_{\mathrm{fl}}\left(K_{\mathrm{mat}}-K_{\mathrm{dry}}\right)^{2}\left(K_{\mathrm{mat}}-K_{\mathrm{fl}}\right)}{\left[K_{\mathrm{mat}}\left(\phi K_{\mathrm{mat}}-\phi K_{\mathrm{fl}}+K_{\mathrm{fl}}\right)-K_{\mathrm{fl}} K_{\mathrm{dry}}\right]^{2}} \\
& =\frac{K_{\mathrm{mat}} K_{\mathrm{fl}}\left(K_{\mathrm{dry}}-K_{\mathrm{mat}}\right)^{2}\left(K_{\mathrm{fl}}-K_{\mathrm{mat}}\right)}{\left[K_{\mathrm{fl}} K_{\mathrm{dry}}-K_{\mathrm{mat}}\left(\phi K_{\mathrm{mat}}-\phi K_{\mathrm{fl}}+K_{\mathrm{fl}}\right)\right]^{2}},
\end{aligned}
$$

which concludes the derivation of equation 16 .
We then find the real root of equation 13 as follows:

$$
K_{\mathrm{sat}}-K_{\mathrm{dry}}-\frac{\left(1-\frac{K_{\mathrm{dry}}}{K_{\mathrm{mat}}}\right)^{2}}{\frac{\phi}{K_{\mathrm{fl}}}+\frac{1-\phi}{K_{\mathrm{mat}}}-\frac{K_{\mathrm{dry}}}{K_{\mathrm{mat}}^{2}}}=0
$$

which provides the single solution $\phi_{1}$ :

$$
\phi_{1}=\frac{K_{\mathrm{fl}}\left(K_{\mathrm{mat}}-K_{\mathrm{dry}}\right)\left(K_{\mathrm{mat}}-K_{\mathrm{sat}}\right)}{K_{\mathrm{mat}}\left(K_{\mathrm{mat}}-K_{\mathrm{fl}}\right)\left(K_{\mathrm{sat}}-K_{\mathrm{dry}}\right)}
$$

for every value $K_{\text {sat }}$.

By combining equations A-2 and A-4, and by assuming that porosity is distributed according to a Gaussian distribution $\phi \sim$ $N\left(\mu_{\phi}, \sigma_{\phi}^{2}\right)$ with mean $\mu_{\phi}$ and variance $\sigma_{\phi}^{2}$, we obtain

$$
f_{K_{\mathrm{sat}}}\left(K_{\mathrm{sat}}\right)=\frac{K_{\mathrm{fl}}\left(K_{\mathrm{dry}}-K_{\mathrm{mat}}\right)^{2}}{K_{\mathrm{mat}}\left(K_{\mathrm{mat}}-K_{\mathrm{fl}}\right)\left(K_{\mathrm{dry}}-K_{\mathrm{sat}}\right)^{2}} \frac{1}{\sqrt{2 \pi \sigma_{\phi}^{2}}} e^{-\frac{\left(\phi_{1}-\mu_{\phi}\right)^{2}}{2 \sigma_{\phi}^{2}}} .
$$

\section{REFERENCES}

Avseth, P., T. Mukerji, A. Jørstad, G. Mavko, and T. Veggeland, 2001, Seismic reservoir mapping from 3-D AVO in a North Sea turbidite system: Geophysics, 66, 1157-1176, doi: 10.1190/1.1487063.

Avseth, P., T. Mukerji, and G. Mavko, 2005, Quantitative seismic interpretation: Cambridge University Press.

Bachrach, R., 2006, Joint estimation of porosity and saturation using stochastic rock physics modeling: Geophysics, 71, no. 5, O53-O63, doi: 10.1190/1.2235991.

Bosch, M., C. Carvajal, J. Rodrigues, A. Torres, M. Aldana, and J. Sierra, 2009, Petrophysical seismic inversion conditioned to well-log data: Methods and application to a gas reservoir: Geophysics, 74, no. 2, O1-O15, doi: $10.1190 / 1.3043796$.

Bosch, M., T. Mukerji, and E. F. Gonzalez, 2010, Seismic inversion for reservoir properties combining statistical rock physics and geostatistics: A review: Geophysics, 75, no. 5, A165-A176, doi: 10.1190/1.3478209.

Brie, A., F. Pampuri, A. F. Marsala, and O. Meazza, 1995, Shear sonic interpretation in gas bearing sands: Presented at SPE Annual Technical Conference and Exhibition.

Buland, A., O. Kolbjørnsen, R. Hauge, O. Skjæveland, and K. Duffaut, 2008, Bayesian lithology and fluid prediction from seismic prestack data: Geophysics, 73, no. 3, C13-C21, doi: 10.1190/1.2842150.

Buland, A., and H. Omre, 2003, Bayesian linearized AVO inversion: Geophysics, 68, 185-198, doi: 10.1190/1.1543206.

Doyen, P., 1988, Porosity from seismic data: A geostatistical approach: Geophysics, 53, 1263-1275, doi: 10.1190/1.1442404.

Doyen, P., 2007, Seismic reservoir characterization: EAGE.

Dvorkin, J., 2008, Yet another $V_{\mathrm{S}}$ equation: Geophysics, 73, no. 2, E35E39, doi: 10.1190/1.2820604.

Dvorkin, J., and A. Nur, 1996, Elasticity of high-porosity sandstones: Theory for two North Sea datasets: Geophysics, 61, 1363-1370, doi 10.1190/1.1444059.

Eidsvik, J., P. Avseth, H. Omre, T. Mukerji, and G. Mavko, 2004, Stochastic reservoir characterization using prestack seismic data: Geophysics, 69 978-993, doi: 10.1190/1.1778241.

Gal, D., J. Dvorkin, and A. Nur, 1998, A physical model for porosity reduction in sandstones: Geophysics, 63, 454-459, doi: 10.1190/1 1444346.

Gallop, J., 2006, Facies probability from mixture distributions with nonstationary impedance errors: 76th Annual International Meeting, SEG Expanded Abstracts, 1801-1805.

González, E. F., T. Mukerji, and G. Mavko, 2008, Seismic inversion combining rock physics and multiple-point geostatistics: Geophysics, 73, no. 1, R11-R21, doi: 10.1190/1.2803748.

Grana, D., and E. Della Rossa, 2010, Probabilistic petrophysical-properties estimation integrating statistical rock physics with seismic inversion: Geophysics, 75, no. 3, O21-O37, doi: 10.1190/1.3386676. 
Gunning, J., and M. Glinsky, 2007, Detection of reservoir quality using Bayesian seismic inversion: Geophysics, 72, no. 3, R37-R49, doi: 10 $.1190 / 1.2713043$.

Hashin, Z., and S. Shtrikman, 1963, A variational approach to the elastic behavior of multiphase materials: Journal of the Mechanics and Physics of Solids, 11, 127-140, doi: 10.1016/0022-5096(63)90060-7.

Karimi, O., H. Omre, and M. Mohammadzadeh, 2010, Bayesian closedskew Gaussian inversion of seismic AVO data for elastic material properties: Geophysics, 75, no. 1, R1-R11, doi: 10.1190/1.3299291.

Kuster, G. T., and M. N. Toksöz, 1974, Velocity and attenuation of seismic waves in two-phase media: Part I. Theoretical formulations: Geophysics, 39, 587-606, doi: 10.1190/1.1440450.

Larsen, A. L., M. Ulvmoen, H. Omre, and A. Buland, 2006, Bayesian lithology/fluid prediction and simulation on the basis of a Markov-chain prior model: Geophysics, 71, no. 5, R69-R78, doi: 10.1190/1.2245469.

Mavko, G., C. Chan, and T. Mukerji, 1995, Fluid substitution: Estimating changes in $V_{\mathrm{P}}$ without knowing $V_{\mathrm{S}}$ : Geophysics, 60, 1750-1755, doi: 10 $.1190 / 1.1443908$.

Mavko, G., and T. Mukerji, 1998, A rock physics strategy for quantifying uncertainty in common hydrocarbon indicators: Geophysics, 63, 19972008, doi: $10.1190 / 1.1444493$.

Mavko, G., T. Mukerji, and J. Dvorkin, 2009, The rock physics handbook: Cambridge University Press.
Mukerji, T., A. Jørstad, P. Avseth, G. Mavko, and J. R. Granli, 2001, Mapping litho-facies and pore-fluid probabilities in a North Sea reservoir: Seismic inversions and statistical rock physics: Geophysics, 66, 9881001, doi: 10.1190/1.1487078.

Nur, A., G. Mavko, J. Dvorkin, and D. Gal, 1995, Critical porosity: The key to relating physical properties to porosity in rocks: 65th Annual International Meeting, SEG, Expanded Abstracts, 878-881.

Papoulis, A., 1984, Probability, random variables and stochastic processes: McGraw-Hill.

Raymer, L. L., E. R. Hunt, and J. S. Gardner, 1980, An improved sonic transit time-to-porosity transform: Presented at 21st Annual Logging Symposium.

Rimstad, K., and H. Omre, 2010, Impact of rock-physics depth trends and Markov random fields on hierarchical Bayesian lithology/fluid prediction: Geophysics, 75, no. 4, R93-R108, doi: 10.1190/1.3463475.

Spikes, K., T. Mukerji, J. Dvorkin, and G. Mavko, 2008, Probabilistic seismic inversion based on rock physics models: Geophysics, 72 no. 5, R87-R97, doi: 10.1190/1.2760162.

Ulvmoen, M., and H. Omre, 2010, Improved resolution in Bayesian lithology/fluid inversion from prestack seismic data and well observations: Part 1 - Methodology: Geophysics, 75, no. 2, R21-R35, doi: 10.1190/1.3294570. 